ARTICLE

DOI: $10.1038 / s 41467-018-06230-0$

OPEN

\title{
Tracing the transitions from pluripotency to germ cell fate with CRISPR screening
}

\author{
Jamie A. Hackett (10) 1,2,3, Yun Huang (1) 1,3, Ufuk Günesdogan 1,3,4, Kristjan A. Gretarsson², \\ Toshihiro Kobayashi, ${ }^{1,3,5} \&$ M. Azim Surani ${ }^{1,3}$
}

Early mammalian development entails transit through naive pluripotency towards postimplantation epiblast, which subsequently gives rise to primordial germ cells (PGC), the founding germline population. To investigate these cell fate transitions, we developed a compound-reporter to track cellular identity in a model of PGC specification (PGC-like cells; PGCLC), and coupled it with genome-wide CRISPR screening. We identify key genes both for exit from pluripotency and for acquisition of PGC fate, and characterise a central role for the transcription regulators $\mathrm{Nr} 5 a 2$ and $Z f p 296$ in germline ontogeny. Abrogation of these genes

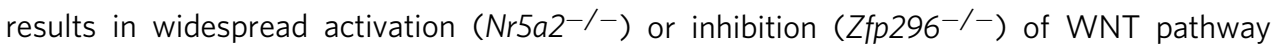
factors in PGCLC. This leads to aberrant upregulation of the somatic programme or failure to activate germline genes, respectively, and consequently loss of germ cell identity. Our study places Zfp296 and Nr5a2 as key components of an expanded PGC gene regulatory network, and outlines a transferable strategy for identifying critical regulators of complex cell fate decisions.

\footnotetext{
${ }^{1}$ Wellcome Trust/Cancer Research UK Gurdon Institute, University of Cambridge, Tennis Court Road, Cambridge CB2 1QN, UK. ${ }^{2}$ Epigenetics and Neurobiology Unit, European Molecular Biology Laboratory (EMBL), via Ramarini 32, 00015 Rome, Italy. ${ }^{3}$ Department of Physiology, Development and Neuroscience, University of Cambridge, Downing Street, Cambridge CB2 3DY, UK. ${ }^{4}$ Department of Developmental Biology, University of Göttingen, Göttingen Center for Molecular Biosciences, Justus-von-Liebig Weg 11, 37077 Göttingen, Germany. ${ }^{5}$ Present address: Center for Genetic Analysis of Behaviour, National Institute for Physiological Sciences, 5-1 Higashiyama Myodaiji, Okazaki, Aichi 444-8787, Japan. These authors contributed equally: Jamie A. Hackett, Yun Huang and Ufuk Günesdogan. Correspondence and requests for materials should be addressed to J.A.H. (email: jamie.hackett@embl.it) or to M.A.S. (email: a.surani@gurdon.cam.ac.uk)
} 
T he germ cell lineage generates the totipotent state and transmits heritable genetic and epigenetic information to the next generation. Robust specification of primordial germ cells (PGC), the precursors of sperm and eggs, is therefore a critical developmental event to ensure the propagation of a species. Approximately 30 specified PGCs are detected in mouse embryos at embryonic day (E) 7.25, which arise from 'competent' epiblast precursor cells in response to BMP and WNT signalling ${ }^{1,2}$. The specification of PGCs is accompanied by induction of key germ-cell genes, repression of the nascent somatic-mesodermal programme, and widespread epigenetic remodelling, including global DNA demethylation ${ }^{3,4}$.

PGC specification follows WNT-dependent induction of the primitive streak/mesodermal gene Brachyury $(T)$, which in mice promotes activation of key PGC specifiers; Blimp1, Prdm14, Cbfa2t2 and $A p 2 \gamma^{5-8}$. These transcription factors form a selfreinforcing network that feeds back to suppress other WNT/ BMP-induced mesodermal genes (including $T$ ), thereby repressing the ongoing somatic programme in early PGCs ${ }^{9}$. Blimp1 and $\operatorname{Prdm} 14$ additionally activate germline-specific genes and initiate epigenome resetting, with mutation of either gene resulting in loss of PGCs by E12.5 ${ }^{10,11}$. The broader gene regulatory network that controls PGC ontogeny has however been relatively uncharted, due to the absence of unbiased functional approaches and the challenges of analysing the limited number of nascent PGCs in embryos.

The recent development of an in vitro model of PGCs, termed PGC-like cells (PGCLC), now facilitates molecular studies of the specification and developmental events of the germ cell lineage ${ }^{12,13}$. PGCLC are derived by inducing naive embryonic stem cells (ESC) that are equivalent to the inner cell mass (ICM) of blastocysts (E3.5-E4.5), towards competent epiblast-like cells (EpiLC), which closely resemble pre-gastrulation mouse epiblast (E5.5-E6.5) ${ }^{14,15}$. EpiLC can in turn be induced to undergo specification as PGCLC in response to BMP and WNT. Specified PGCLC are equivalent to migratory PGCs in vivo (E8.5-E10.5) ${ }^{12}$, and have the potential to develop to mature functional gametes ${ }^{13,16}$. This model is therefore appropriate to investigate the inherent regulatory mechanisms of nascent germ cells, and the preceding developmental transitions from ICM, through postimplantation development and PGC specification.

The advent of genome-wide CRISPR screening has enabled unbiased interrogation of recessive gene function in a wide spectrum of biological contexts ${ }^{17-19}$. We reasoned that by designing appropriate reporters, CRISPR screening could be adapted to identify genes involved in controlling sequential cell fate decisions during lineage-specific differentiation. Specifically, by employing the PGCLC model we embarked on identification of genes that are important for (i) maintenance of naive pluripotency, (ii) transition to the germline competent state (EpiLC) and, (iii) specification of the PGC lineage. We further investigate novel candidates to reveal their key role and mechanistic function during nascent germ cell development. From a broader perspective, we demonstrate that unbiased CRISPR screening can be adapted to probe the genetic basis of complex multi-step developmental processes, using the germline lineage as a paradigm.

\section{Results}

Compound reporter for developmental transitions to PGC fate. We set out to design a reporter system that can distinguish between successive cell identities during the developmental transitions from naive pluripotency to specified PGC fate. Single-cell RNA-seq data revealed that Stella (also known as Dppa3) is expressed in the naive pluripotent ICM but is rapidly downregulated in post-implanation epiblast, with re-activation occurring specifically in nascent PGCs (from E7.5) ${ }^{20}$. In contrast Esg1 (also known as Dppa5) is also expressed in the ICM, but maintains high expression during post-implanation development, and subsequently becomes strongly downregulated in early PGCs; with low germline expression reacquired later (>E9.5) (Fig. 1a). To exploit the mutually exclusive expression of these genes, we generated an ESC line with compound Stella-GFP and Esg1-tdTomato (SGET) reporters. In this 'traffic-light' system naive pluripotent cells are double-positive (yellow), post-implantation epiblast cells are Esg1-positive (red) and nascent PGCs are Stella-positive (green). We monitored SGET expression during mouse development by tetraploid complementation and chimera formation, and observed strong double-activation in naive pluripotent epiblast at E4.5 (Fig. 1b). This resolved to single Esg1-tdTomato activity in E6.0 epiblast, with both reporters subsequently silenced in somatic tissues by E7.0. Importantly Stella-GFP is specifically reactivated in PGCs by E8.5, until E12.5, with Esg1-tdTomato additionally showing weak expression in later PGC stages (Fig. 1b). Thus, SGET faithfully recapitulates expression of the endogenous genes during the transitions towards PGC fate.

Next, we examined SGET activity during in vitro specification of PGCLC, which initiates from naive ESC and transitions through 'competent' EpiLC ${ }^{12}$. SGET ESC were largely doublepositive $\left(\mathrm{SG}^{+} \mathrm{ET}^{+}\right)$, but resolved to Esg1-only expression $\left(\mathrm{SG}^{-} \mathrm{ET}^{+}\right)$in $>97 \%$ of cells upon induction into EpiLC. In contrast, nascent PGCLC (day 2) reactivated Stella while concomitantly repressing Esgl $\left(\mathrm{SG}^{+} \mathrm{ET}^{-}\right)$, with later stage PGCLC (day 6) exhibiting low Esg1 expression ( $\mathrm{SG}^{+} \mathrm{ET}^{\mathrm{low}}$ ) (Fig. 1c). PGCLC carrying SGET therefore recapitulate the in vivo dynamics. To functionally validate the SGET reporter system, we generated ESC with a mutation in the key PGC-specifier Blimp1 (Supplementary Fig 1A), which resulted in a significant reduction (up to 2.8-fold) in the efficiency of PGCLC generation (Fig. 1d; Supplementary Fig 1B). The remaining Blimp $1^{-1-}$ PGCLC exhibited aberrant gene expression (Supplementary Fig 1C), consistent with in vivo studies where a fraction of nascent 'PGCs' remain but with an altered transcriptome ${ }^{10}$. The SGET reporter system is thus ideal for monitoring successive changes in cell identity, from naive ESC to specified PGCLC.

CRISPR screen identifies key genes for naive ESC. We introduced a single-copy of Cas 9 into SGET ESC (Supplementary Fig 1D), and subsequently infected this line with an integrating lentiviral library of exon-targeting guide RNAs (gRNA) ${ }^{21}$, in independent biological replicates. Statistical analysis of gRNA frequency in the ESC population, relative to the initial frequency, reveals essential genes because their cognate gRNAs become depleted concomitant with cell loss. We reasoned that by flow sorting SGET cells that successfully acquire each sequential fate during PGCLC specification, we could identify essential genes for each transition by comparing gRNA frequency to the preceding cell population (Supplementary Fig 1E). Accordingly, this approach filters out genes with a role in prior fate transitions and reveals the critical regulators for each stage of multi-step differentiation events, based on functional requirement.

Following introduction of the CRISPR library, we identified 627 genes that lead to a loss of naive ESC maintained in 2i/LIF when knocked-out $(p<0.01$ in all independent replicates using the relative ranking algorithm (RRA) in the MAGeCK tool 22 ) (Fig. 2a). Gene ontology (GO) terms for these genes were highly enriched for fundamental biological processes such as ribosome biogenesis (Bonferroni adjusted $p=2.88 \times 10^{-27}$; e.g. Rps5) and protein translation (Bonferroni adjusted $p=4.25 \times 10^{-30}$; e.g. Eif6), supporting the efficacy of the library (Supplementary Fig 2A). Notably ESC carrying mutations for the core 
a

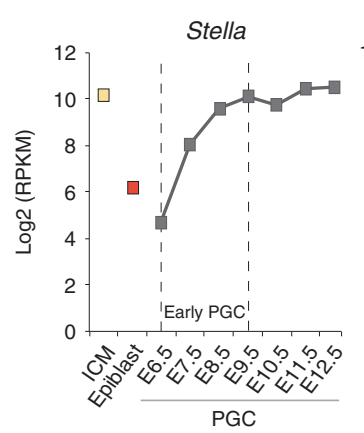

b

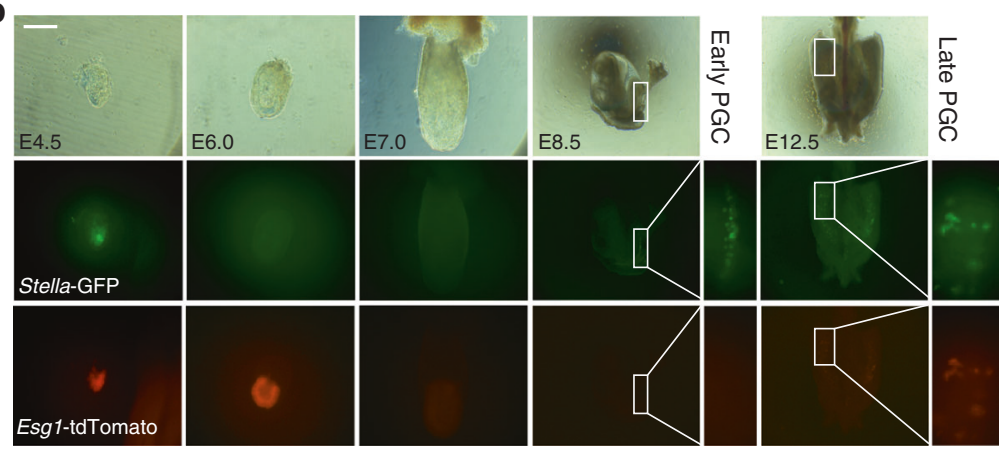

C

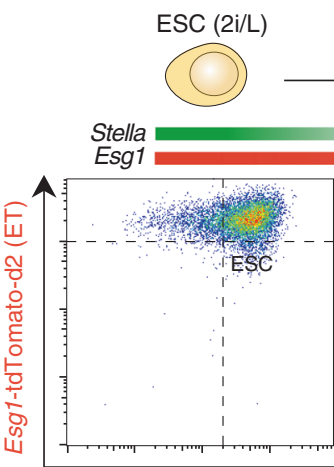

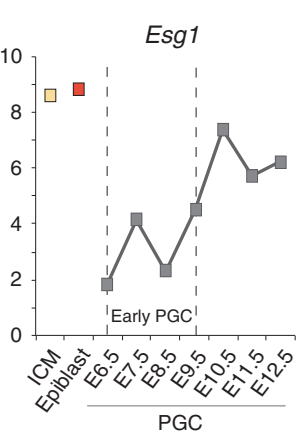

EpiLC

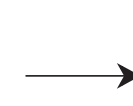

d2 PGCLC
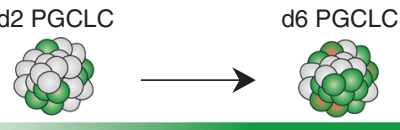

PGCLC
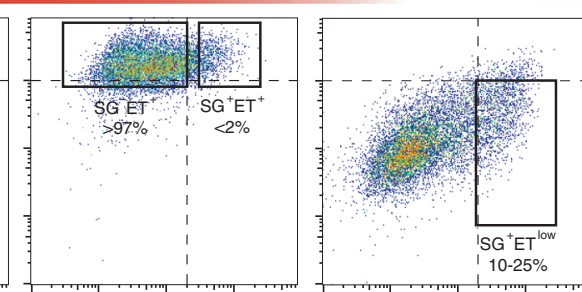

Stella-GFP (SG)

$d$

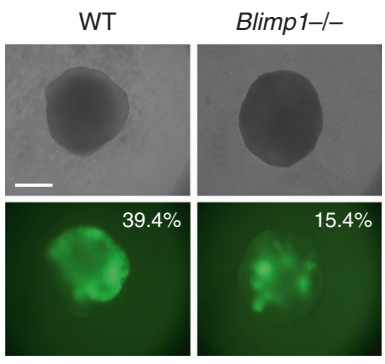

Fig. 1 The SGET reporter for tracking cell fate transitions toward PGCs. a Single-cell RNA-seq analysis showing reciprocal gene expression patterns of endogenous Stella and Esg1 during early development (ICM; E3.5 or Epiblast; E6.5) and in primordial germ cells (PGC). b Chimera and tetraploid complementation assays confirm faithful expression of the Stella-GFP (green) and Esg1-tdTomato (red) SGET reporter during early development and in PGCs. c Representative FACS analysis of SGET activation during in vitro cell fate transitions of ESC into EpiLC and subsequently PGCLC. d Representative images of impaired PGCLC induction from Blimp1-/- SGET ESC. Numbers indicate percentage of SG+ETlow PGCLC at day 5 as determined by FACS. Scale $\operatorname{bar}(\mathrm{s}): 200 \mu \mathrm{m}$

pluripotency factors Oct4 and Sox 2 were also highly depleted, consistent with their essential role in propagating pluripotent $\mathrm{ESC}^{23}$ (Fig. 2a, b; Supplementary 2B). The majority of naive pluripotency genes were not depleted however, which is in line with the capacity of $2 \mathrm{i} / \mathrm{LIF}$ media to buffer against a single genetic perturbation to the naive pluripotency network (Fig. 2b) ${ }^{24}$. Importantly, this enables the role of such naive pluripotency genes to be examined in PGC specification, where they are also typically expressed, since knockout cells remain in the population. Exceptions however are Nanog and Tfcp2l1, which are likely depleted owing to a proliferation disadvantage upon knockout in ESC. In contrast to the many depleted genes, we observed that only 21 genes become enriched in the population upon knockout, primarily corresponding to tumour suppressors (Supplementary Fig 2B \& C). Among these $\operatorname{Trp53}(p 53)$ is the top hit, suggesting that $p 53$-mutant mouse ESC acquire a major selective advantage, similar to recent reports in human ESC ${ }^{25}$.

We next compared the genes essential for naive ESC (in $2 \mathrm{i} / \mathrm{LIF}$ ) with those essential for ESC in conventional serum/LIF culture, which represents an alternative pluripotent state 23 . Using datasets from the same CRISPR library ${ }^{21}$, we observed good overlap (62\%) between ESC conditions, but noted $38 \%$ of genes appear to be critical under only specific pluripotent culture parameters (Fig. 2c). Among the 2i/LIF specific genes were $O g d h$ and Dlst; two enzymes that mediate a-ketoglutarate metabolism which feeds into the TCA cycle. We used siRNAs to test acute loss of Ogdh and observed a significant reduction of proliferation and/or increased cell death in 2i/LIF ESC but not serum/LIF ESC (Fig. 2d). We also tested Txn1, which was scored as essential in both pluripotent ESC states, and found an equivalent cell reduction in $2 \mathrm{i} / \mathrm{LIF}$ and serum/LIF (Fig. 2d). These data suggest ESC in distinct pluripotent states rely, in part, on distinct genetic networks, with ESC in $2 \mathrm{i} / \mathrm{L}$ uniquely reliant on $O g d h$ metabolism of a-ketoglutarate for example. More generally, establishing this mutant SGET ESC population enables screening for functional regulators of EpiLC, and subsequently PGCLC, without confounding general survival genes.

Acquisition of post-implantation epiblast fate. We next used fluorescence-activated cell sorting (FACS) to isolate EpiLC that successfully transited to the $\mathrm{SG}^{-} \mathrm{ET}^{+}$epiblast state, as well as cells that maintained Stella expression $\left(\mathrm{SG}^{+} \mathrm{ET}^{+}\right)(<2 \%)$, indicative of failure to exit naive pluripotency. Analysis of this $\mathrm{SG}^{+} \mathrm{ET}^{+}$EpiLC population revealed enrichment for 21 candidate genes with potential intrinsic roles in dissolution of naive pluripotency $(p<$ 0.01 using the $\mathrm{RRA}^{22}$ ), including the two prototypical regulators $T c f 3$ and $Z f p 281$ (Fig. 2e; Supplementary Fig 3A) ${ }^{26,27}$. The candidates additionally included the epigenetic regulator Dnmt1 and also Rest, which are known to be important during early differentiation 28,29 .

To test the role of novel candidates in driving exit from pluripotency we generated ESC knockouts for Zmym2 (also known as $Z f p 198)$, FoxP1, Uchl5 and $Z f p 281$ as a positive control, using CRISPR targeting. Consistent with their failure to repress Stella-GFP during EpiLC formation, mutant EpiLC were also impaired in activation of key epiblast markers Fgf5, Dnmt3b and Otx2, while their proliferation appeared unaffected (Fig. 2f; Supplementary Fig 3B-C). We observed both a delay in activation of epiblast genes and a reduction in their absolute levels in 

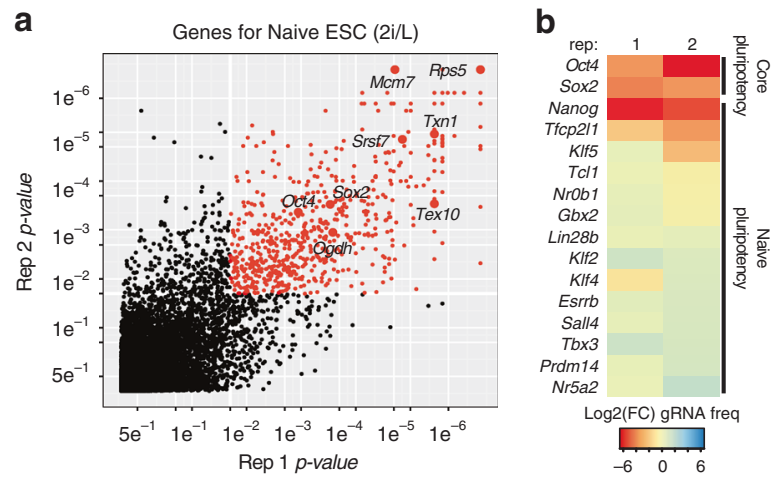

C

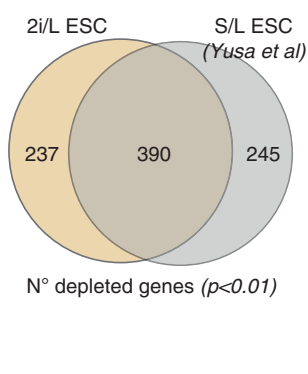

d

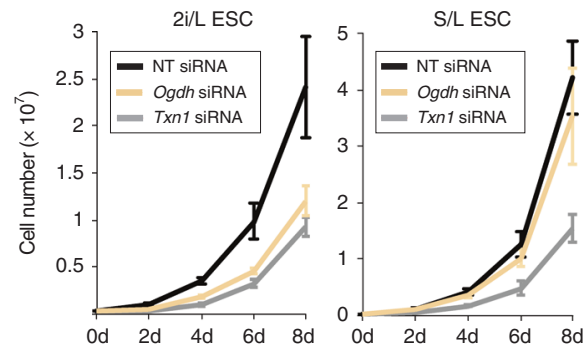

e

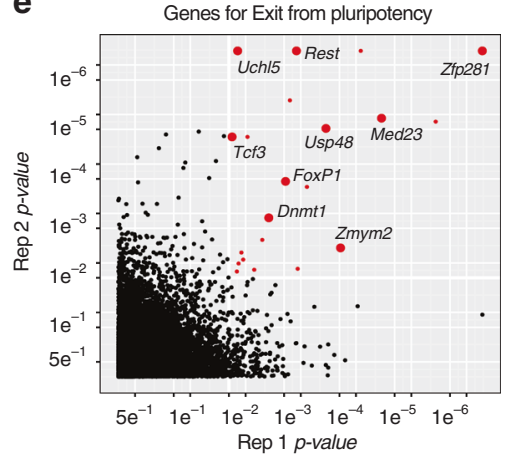

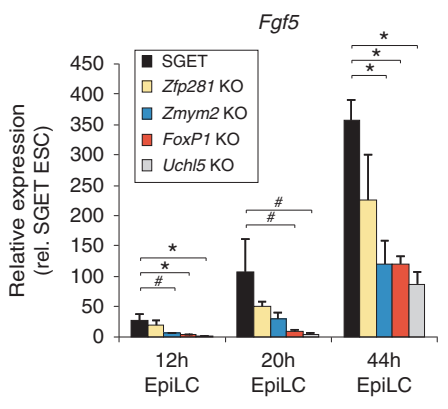

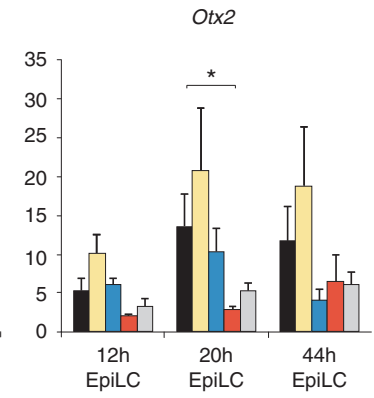

Fig. 2 Identification of important genes for ESC and EpiLC induction. a Scatter plot showing essential genes for ESC propagation in 2i/LIF (red datapoints) as determined by a CRISPR screen. $\mathbf{b}$ Heatmap showing mean fold change (FC) in normalised frequency of gRNAs targeting core- and naive- pluripotency genes in ESC relative to frequency in starting gRNA library. Reduced frequency indicates a functionally important gene for ESC in 2i/LIF. c Venn diagram intersecting significant depleted genes in 2i/LIF ESC (2i/L) with serum/LIF (S/L) maintained ESC. d Proliferation of ESC following siRNA knockdown of Ogdh or Txn1 in ESC maintained in 2i/L or S/L culture conditions. e Scatter plot showing significantly enriched gene knockouts in SG+ET+ EpiLC that have failed to exit naive pluripotency. $\mathbf{f}$ Gene expression showing impaired activation of key epiblast markers in EpiLC carrying knockouts for candidate exit from pluripotency regulators. Significance was determined using a one-tailed $t$-test; ${ }^{*} p<0.05$; ${ }^{\#} p<0.1$. Error bars show s.e.m. of triplicate independent experiments

knockout lines, despite high levels of powerful differentiationinducing FGF and ACTIVIN in the culture medium. Notably, the Zmym2-, FoxP1-, Uchl5- mutant lines exhibited expression defects comparable with the bona fide exit from pluripotency regulator Zfp281, supporting an important role for them in the timely acquisition of post-implantation cell identity. Consistently all three candidates are transcriptionally upregulated during EpiLC induction (Supplementary Fig 3D).

Candidate genes for specification of PGC fate. We next focused on genes involved in mouse germline specification by inducing PGCLC from competent $\mathrm{SG}^{-} \mathrm{ET}^{+}$EpiLC. In order to obtain sufficient numbers of PGCLC for coverage of the gRNA library, we optimised and scaled-up the induction (Supplementary Fig $4 \mathrm{~A}$ ). Specified $\mathrm{SG}^{+} \mathrm{ET}^{\text {low }}$ PGCLC were FACS purified at day (d) 6 of differentiation to allow time for depletion of cells carrying mutations in critical germline genes. Because PGCLC numbers remained a limiting factor however, we relaxed the candidate threshold to $p<0.05$, while retaining a $p<0.01$ in at least one replicate, to account for increased noise (Supplementary Fig 4B). This resulted in identification of 23 candidate genes involved in specification and/or development of nascent PGC. The frequency of gRNAs targeting these genes was highly depleted in PGCLC relative to preceding EpiLC (Fig. 3a), while negative control gene families were not depleted (Supplementary Fig 4C). Moreover, genes with an established critical role in PGC specification, such as Blimp1, Cbfat2t2 and Prdm14, also showed marked reduction in gRNA frequency in PGCLC, supporting the efficacy of the screen (Fig. 3a). These genes narrowly failed to meet significance across all replicates however; Cbfa2t2 scored $p$-values of $p=$ 0.00006 and $p=0.22$ for example. We also noted that several pluripotency genes (Nr5a2, Esrrb and Sall4) were depleted specifically from PGCLC (Supplementary Fig 4C), suggesting that some genes typically linked with pluripotency have a potentially important function in the germline.

To refine our candidate list we examined their expression profile during induction of PGCLC from the transduced SGET ESC by RNA-seq. This confirmed that $\mathrm{SG}^{+} \mathrm{ET}^{\text {low }}$ PGCLC activate high levels of key PGC markers such as Blimp1, Stella, Itgb3, Nanos3 and Prdm14, as well as repressed Uhrf1 and Klf4, supporting their authentic germline identity (Supplementary Fig 4D). The dynamics of candidate gene expression revealed four broad clusters, with cluster 1 and 4 corresponding to genes that are expressed relatively stably throughout ESC to PGCLC transition; the latter is nonetheless only just above the detection threshold implying it may filter out false-positives. In contrast, cluster 2 and 3 reflected dynamically regulated genes that are activated in PGCLC, with cluster 3 corresponding to genes fully silenced in EpiLC before strong germ cell re-activation, and including known PGC specifiers (Fig. 3b). From these, we chose to focus on Zfp296 and Nr5a2, since they rank 3 and 7 overall in the screen, and exhibit comparable PGCLC expression dynamics to Prdm14 and Blimp1 (Fig. 3b; Supplementary Fig 4D). Nr5a2 encodes an orphan nuclear receptor previously associated with reprogramming towards pluripotency, and also has a role in extra embryonic development, which leads to embryonic lethality when 

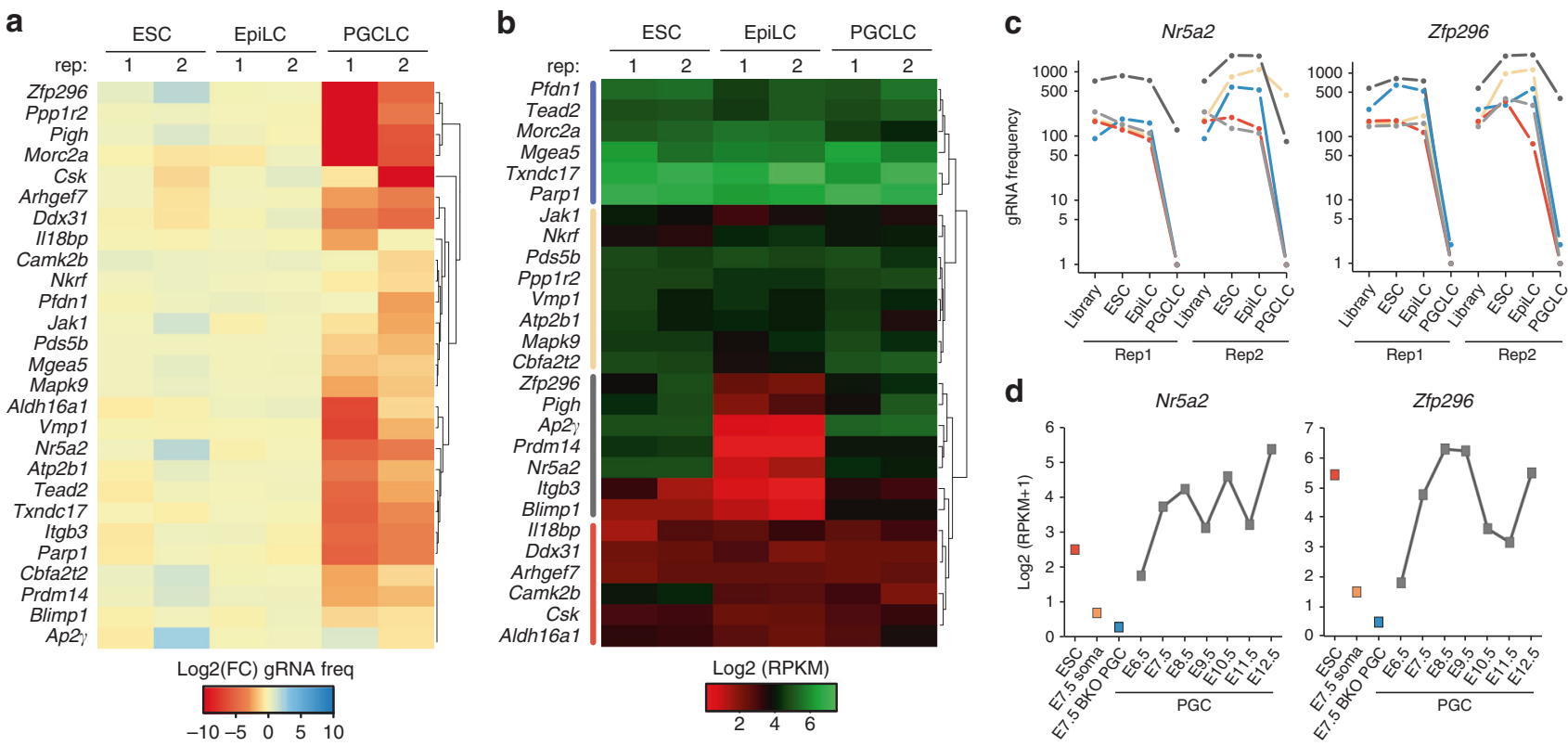

Fig. 3 Candidate genes for primordial germ cell fate. a Heatmap showing mean fold-change (FC) in normalised frequency of gRNAs that target candidate PGC regulators, relative to their frequency in the preceding cell state. $\mathbf{b} \log _{2}$ RPKM gene expression dynamics of candidate PGC regulators during

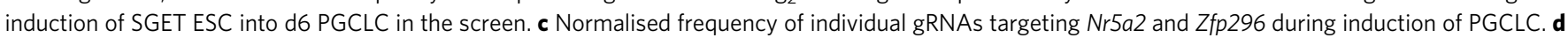
Expression dynamics of Nr5a2 and Zfp296 during in vivo formation of PGCs by single-cell RNA-seq. Blimp1-/- PGC (BKO) and soma are shown for reference

abrogated ${ }^{30} . Z f p 296$ encodes a zinc-finger protein that was among the original 20 Yamanaka factors, and has recently been shown to influence heterochromatin and induced pluripotent stem cell (iPSC) generation ${ }^{31-33}$.

Inspection of the individual gRNAs that target Nr5a2 and Zfp296 revealed a strong and reproducible reduction of their frequency specifically in PGCLC, but not in the preceding developmental transitions (Fig. 3c). Moreover, single-cell RNAseq indicates both genes are highly upregulated in nascent PGC in vivo at the very onset of specification, but not in adjacent somatic cells (Fig. 3d). Both Nr5a2 and Zfp296 are also strongly upregulated in early human PGCs (Supplementary Fig 4E), and fail to be activated in Blimp $1^{-1-}$ PGC that are destined to diverge from the germline lineage (Fig. $3 \mathrm{~d}$ ). The striking upregulation of these genes during incipient PGC specification, coupled with their high rank in the functional screen, prompted us to investigate their role in detail.

Nr5a2 and Zfp296 regulate germline ontogeny. We used CRISPR editing to generate frame-shifted null-alleles of $\mathrm{Nr} 5 \mathrm{a} 2$ or Zfp296 in SGET ESC. Nr5a2-/- ESC proliferated normally, expressed comparable levels of naive pluripotency genes, and exhibited indistinguishable morphology from parental and matched WT ESC derived from the same targeting process. However upon induction of PGCLC, we observed a significant reduction in specification efficiency of $\mathrm{Nr} 5 \mathrm{a} 2^{-/-}$cells at d2 relative to WT controls (WT 19.8\% \pm 8.9 ; KO 6.4\% \pm 2.3 ) (Fig. 4a). The impaired induction of Nr5a2-mutant cells was further reflected in d6 PGCLC, and across multiple independent knockout lines (WT $44.7 \% \pm 4.0$; KO $17.4 \% \pm 4.6$ ), suggesting an important role for Nr5a2 in PGC specification (Fig. 4a; Supplementary Fig 5A). We next tested $Z f p 296^{-/-}$ESC lines, which also exhibited normal morphology and pluripotent gene expression profiles, albeit with modestly reduced proliferation rate. Upon induction, PGCLCs were specified from Zfp296-mutant cells at apparently normal efficiency at $\mathrm{d} 2$ (WT $14.9 \% \pm 3.2$; KO $16.2 \% \pm 2.2$ ), as judged by the SGET reporter. However, by d6 we observed a highly significant depletion of PGCLC in multiple mutant lines (WT $43.1 \%$ \pm 3.5 ; KO $8.9 \% \pm 2.8$ ), suggesting the effects of $Z f p 296$ abrogation on PGCLC are progressive and manifest after specification (Fig. 4b). More generally, these data indicate that loss-of-function of either $N r 5 a 2$ or $Z f p 296$ leads to a significant impairment of germ cell development.

Despite the dramatic reduction in numbers, both mutant lines give rise to some putative PGCLC. To examine the identity of these cells, and their developmental trajectory, we performed RNA-seq. Unbiased hierarchical clustering revealed that $N r 5 a 2^{-I-}$ EpiLC were interspersed with WT, but that PGCLC were clustered according to genotype, implying transcriptional differences in the absence of Nr5a2 primarily arise upon induction of germline fate (Supplementary Fig 5). To investigate this further we used principal component analysis (PCA), which confirmed that mutant EpiLC cluster closely with WT but that Nr5a2-null PGCLC progressively diverge from WT controls during their development (Fig. 4c). By d6 a strong distinction is apparent. This is consistent with $\mathrm{Nr} 5 \mathrm{a} 2^{-/-}$EpiLC being competent for the germline lineage but undergoing an abnormal developmental trajectory upon PGCLC specification, which coincides with the timing of Nr5a2 upregulation in WT cells.

Global transcriptome clustering of $Z f p 296-$ null cells indicated that precursor EpiLC were distinct yet highly comparable between WT and knockout lines (Supplementary Fig 5). Indeed, PCA showed Zfp296-/- EpiLC cluster closely with WT. In contrast, $\mathrm{d} 2$ mutant PGCLC exhibited a significantly different state from WT counterparts, that is closer to precursor EpiLC and thus apparently impaired in its developmental path towards germline fate, which is consistent with the subsequent loss of PGCLC numbers (Fig. 4d). The remaining Zfp296-mutant PGCLC at d6 appeared to partially re-establish the expected global profile, albeit with greater transcriptome variance between lines than WT. This implies that despite widespread 
a

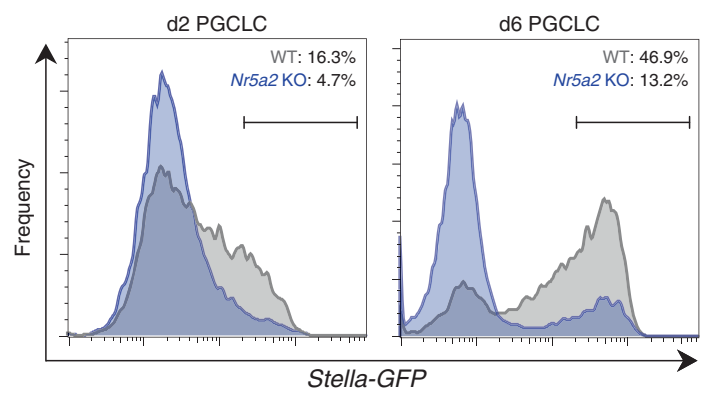

b

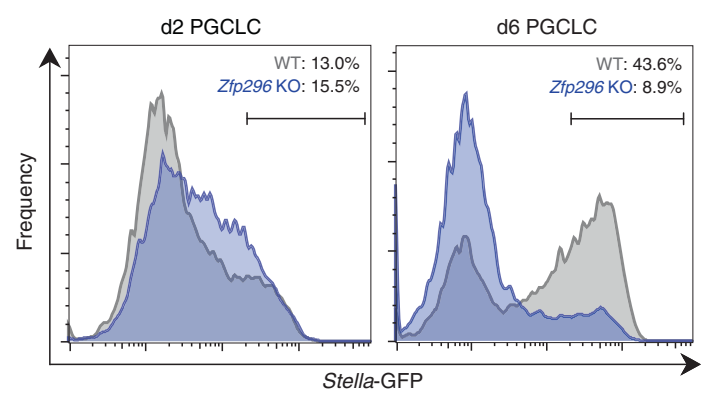

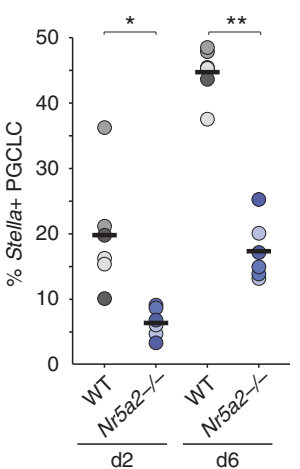

C
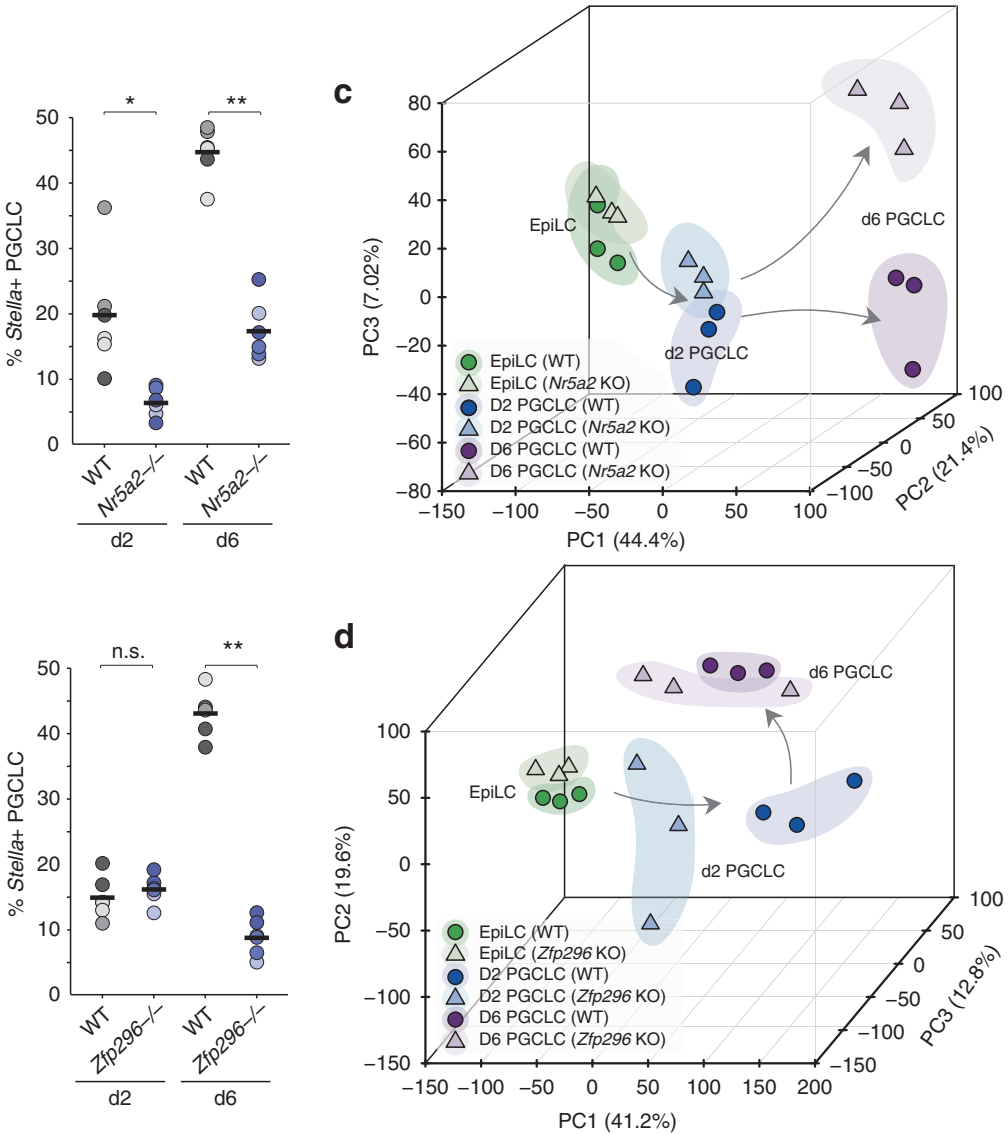

Fig. 4 Nr5a2 and Zfp296 are key regulators of germ cell development. a Representative FACS plot showing impaired induction of PGCLC in Nr5a2-knockout (KO) cells. Shown right is replicate quantifications of independent WT and KO lines. Independent lines are colour-matched. Bar indicates mean value. $\mathbf{b}$ Representative FACS of PGCLC in Zfp296-knockout SGETs, with replicate quantification shown right. c Principal component analysis (PCA) showing the developmental trajectory of independent $\mathrm{Nr}_{\mathrm{Sa}} \mathrm{2}^{-/-}$and matched-WT SGET lines during induction of PGCLC, based on global transcriptome. $\mathbf{d}$ PCA of independent Zfp296- $/-$ and matched-WT SGET transcriptomes. ${ }^{\star} p<0.05 ;{ }^{*} p<0.01$

transcriptional mis-regulation, a minor proportion of mutant PGCLC can overcome the effects of $Z f p 296$ abrogation, and give rise to putative germ cells (Fig. $4 \mathrm{~d}$ ). Overall, these data suggest that loss of Nr5a2 or Zfp296 has a dramatic influence on PGC development.

Nr5a2 and Zfp296 converge on reciprocal regulation of WNT. To investigate the mechanisms that impair development of mutant PGCLC and underpin their aberrant transcriptomes in more depth, we identified differentially expressed genes (adjusted $p<0.05 ;>2$ FC) in EpiLC and PGCLC. This revealed that downregulation of naive pluripotency genes and upregulation of post-implantation markers proceeded similarly between $N r 5 a 2^{-1-}$ and WT EpiLC counterparts (Fig. 5a). Moreover, there was appropriate upregulation of early PGC markers such as Nanos3, Stella, Blimp1 and Prdm14 in d2 Nr5a2-/- PGCLC, that was at least equivalent to WT, albeit with a modest failure to upregulate Nanog. However, we noted d2 Nr5a2-null PGCLC exhibited a highly significant overexpression of WNT pathway genes and targets, including $C d x 2, T$ and Mixl1 (Fig. 5a). This was further reflected in gene ontology (GO) analysis of differentially expressed genes at $\mathrm{d} 2$, which highlighted canonical WNT signalling pathway (Bonferroni adjusted $p=0.017$ ) and pattern specification process (Bonferroni adjusted $p=0.014$ ) as top hits (Table S1). We confirmed hyperactivation of key WNT pathway genes $T$ and $W n t 3$ in early Nr5a2-l- PGCLC using qRT-PCR on independent replicates (Fig. 5b). Strong overexpression of the master mesoderm regulator $T$ is predicted to override its role in germ cell induction and divert cells toward a somatic mesendoderm programme. Consistently, the surviving Nr5a2 ${ }^{-/-}$PGCLC at $\mathrm{d} 6$ exhibit acute activation of mesendoderm regulators such as Tbx4, Hey1 and Pou2f3 (Fig. 5a). This collectively suggests that the absence of Nr5a2 leads to aberrant transduction of WNT signalling in nascent PGCLC, which in turn leads to upregulation of mesendoderm master-regulators and consequently derepression of a somatic programme. It is possible that the aberrant activation of somatic genes in the absence of Nr5a2 is a contributing factor that drives the majority of prospective PGCLC out of the germline programme, leading to reduced efficiency of specification.

We next analysed gene expression in Zfp296-null EpiLC, which revealed that pluripotency genes including Nanog, Esrrb and Tfcp $2 l 1$ were repressed as expected, while EpiLC markers such as Fgf5 and Dnmt3b were appropriately upregulated. In contrast mutant PGCLC at $\mathrm{d} 2$ exhibited a clear signature of mis-expressed genes (Fig. 5a). Remarkably, GO analysis again suggested this primarily corresponded to WNT targets, but in contrast to hyperactivation of WNT in Nr5a2-1- PGCLC, Zfp296-null PGCLC exhibited a severe block of WNT-associated gene activation; the top enrichment is negative regulation of canonical WNT signalling (Bonferroni adjusted $p=0.0064$ ). Among all genes $T$ is the most significantly downregulated in early Zfp296mutant PGCLC, with Cdx2, Notum and Dkk1 also being among 


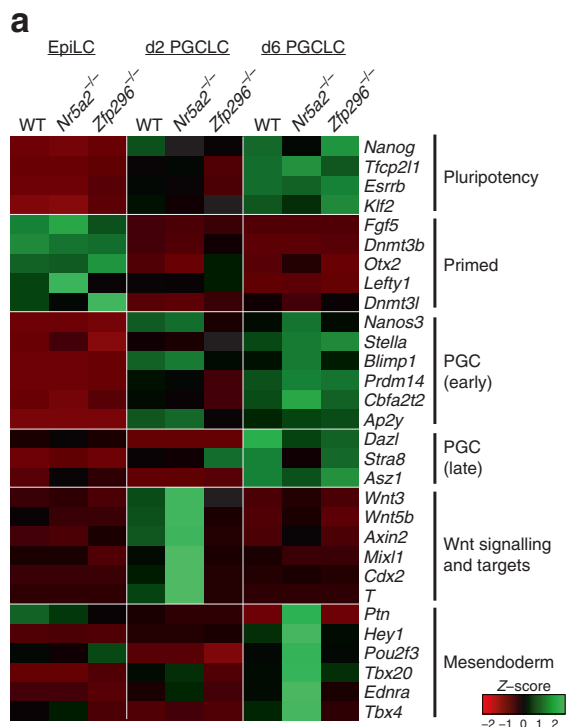

b
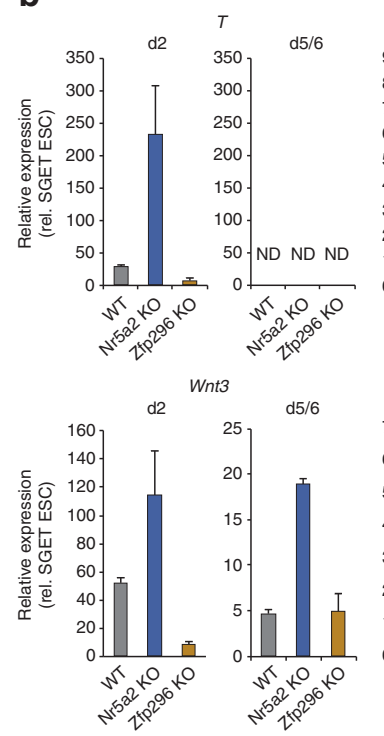
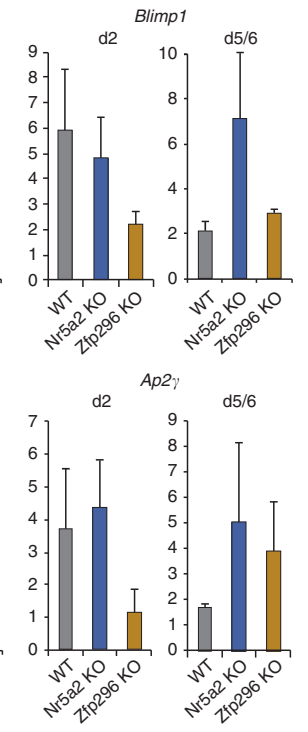
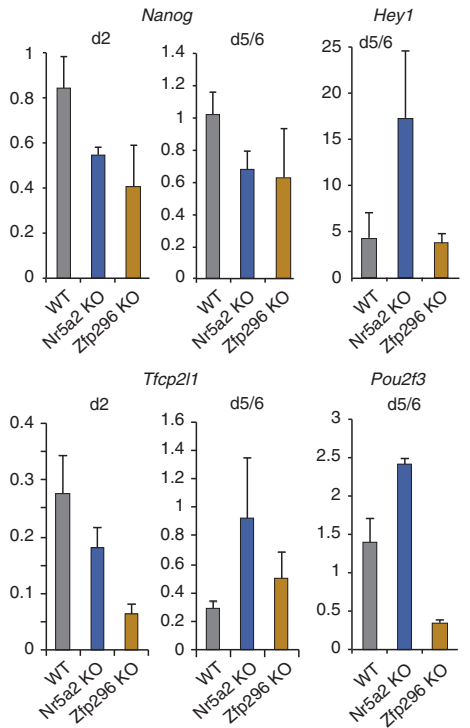

Fig. 5 Compound mis-regulation of WNT and germ cell genes in the absence of Nr5a2 and Zfp296. a Heatmap showing change in mean expression profile of selected genes from triplicate independent WT, Nr5a2-/- or Zfp296-/- lines during PGCLC induction by RNA-seq. b Quantitative validation of gene expression changes in PGCLC from WT, Nr5a2-/- and $Z f p 296^{-/-}$lines by independent qRT-PCR experiments. Error bars show s.e.m. of duplicate biological experiments, each comprising at least twelve independent replicate inductions. ND not detected

the top 20. The absence of appropriate level WNT signalling could manifest as impaired activation of critical germline genes that are WNT and/or $T$ targets $^{5}$. Indeed, Zfp296-null PGCLC at d2 exhibited a block in Blimp1, Prdm14 Cbfa2t2a and $A p 2 \gamma$ activation (Fig. 5a). qRT-PCR analysis independently confirmed highly impaired T and WNT genes in Zfp296-mutant PGCLC and concomitant significant reduced activation of germline regulators Blimp1 and Ap2 $\gamma$ (Fig. 5b). This suggests that absence of $Z f p 296$ establishes a reciprocal developmental failure relative to deletion of Nr5a2, with both genes converging on regulation of appropriate WNT signalling in nascent PGCLC. In the case of $Z f p 296$, this appears to manifest in a delayed but dramatic loss of PGCLC by d6. Notably, the $\mathrm{SG}^{-} \mathrm{ET}^{-}$cells from both $\mathrm{Nr} 5 \mathrm{a} 2$ and Zfp296 embryoids, which have putatively acquired somatic fate, exhibited comparable expression of lineage-specific markers Sox17, Hoxa1 and Sox7 relative to wild type (Supplementary Fig 6A). This implies that the loss of function for both genes does not impede the exit from the pluripotent state towards somatic fates, but these cells are impaired specifically in their capacity to form PGCs.

Rescue of Nr5a2 and Zfp296 deficiency. We considered that the impaired specification and aberrant transcriptome of mutant PGCs could be due to impaired epigenetic reprogramming, as reported in $\mathrm{Prdm} 14^{-/}-\mathrm{PGCs}^{11}$. To investigate this, we quantified global DNA methylation erasure and observed that WT, Nr5a2 $2^{-/-}$and $Z f p 296^{-/-}$PGCLC all underwent extensive and comparable DNA demethylation (Fig. 6a). This was confirmed by bisulfite pyrosequencing analysis of LINE-1 elements (Supplementary Fig 6B). We therefore turned our attention back to understanding whether the observed mis-regulation of WNT could be driving impaired germline fate by exposing incipient PGCLC to WNT inhibitor (WNTin: XAV939) or WNT agonist (WNTag: Chiron). Addition of WNTin led to strong downregulation of key PGC genes including $T$ and $W n t 3$, as well as Nanog, consistent with the effects of impaired WNT transduction in Zfp296-null PGCLC (Fig. 6b). Reciprocally, WNTag affected an increase in expression of $T$ and Wnt3 at $\mathrm{d} 2$, while also downregulating Nanog, which is in line with the effects of WNT over- activity in Nr5a2-null PGCLC. Indeed, WNTag also elicited overexpression of mesendoderm gene Hey1 by d6 PGCLC (Fig. 6c). Notably WNTinh led to a significant depletion of specified PGCLC $(p<0.05)$, while partial WNT activation by WNTag also trended towards impaired PGCLC specification (Fig. 6d). These data suggest that precise levels of WNT activity are necessary for optimal PGC specification.

Addition of WNTin to Nr5a2-/- PGCLC rescued hyperactivated $T$ and $W n t 3$, demonstrating that their upregulation in Nr5a2-mutant PGCLC is likely a direct consequence of deregulated WNT activity (Fig. 6b). In contrast addition of excess WNTag to Zfp296-mutant PGCLC only resulted in modest rescue of aberrantly repressed WNT targets, suggesting that in the absence of $Z f p 296$, germ cells cannot respond to WNT activation, even when forced. We therefore attempted to rescue the specification defects by generating mutant PGCLC that carry Doxycycline (DOX)-inducible expression of wild-type Nr5a2 and Zfp296. We induced Nr5a2 expression specifically during PGCLC induction in a $N r 5 a 2^{-/-}$background, which led to a highly significant rescue of PGCLC specification. Moreover, this reimposed suppression of somatic and WNT target genes, implying a direct effect of $\mathrm{Nr} 5 \mathrm{a} 2$ on these pathways (Fig. 6e; Supplementary Fig 6C-D). In contrast, we found expression of Zfp296 to be highly toxic to both ESC and PGCLC, even at very low background (leaky) levels, suggesting precise control over its expression level is important.

Remarkably DOX-induced Nr5a2 expression in mutant PGCLC resulted in increased PGCLC induction as compared to wild-type counterparts. This encouraged us to test whether Nr5a2 could drive germline fate independently of BMP cytokines that are normally requisite. Here, forced expression of $\mathrm{Nr} 5 \mathrm{a} 2$ in wild-type EpiLC led to emergence of $\mathrm{SG}^{+} \mathrm{ET}^{\text {low }}$ PGCLC at >tenfold higher efficiency than background; at levels comparable with forced expression of canonical PGC-specifier $\operatorname{Prdm} 14$, albeit at low absolute frequency (3.3\%) (Fig. 6f). Induction of Nr5a2 resulted in PGCLC with apparently similar expression profiles as authentic PGCs (Supplementary Fig 6E). This indicates that activation of $N r 5 a 2$ can, at low efficiency, directly induce PGC-like fate from competent EpiLC. More 

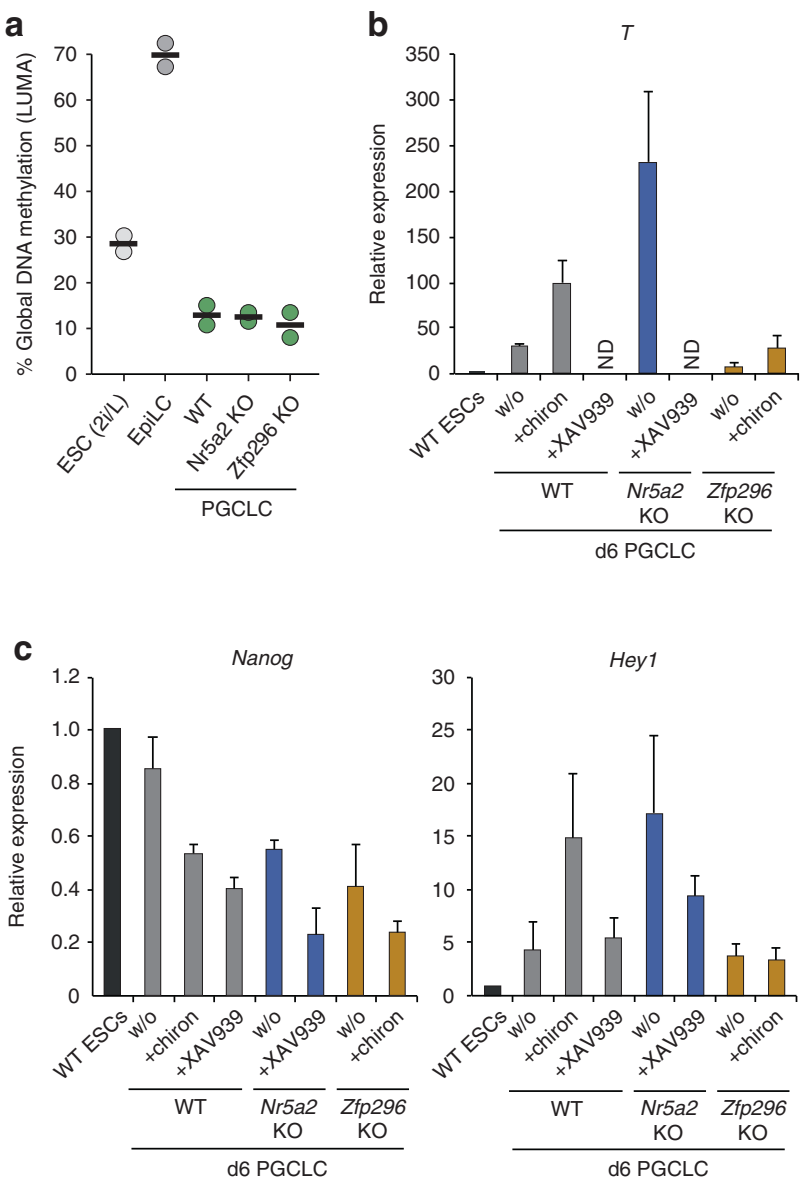

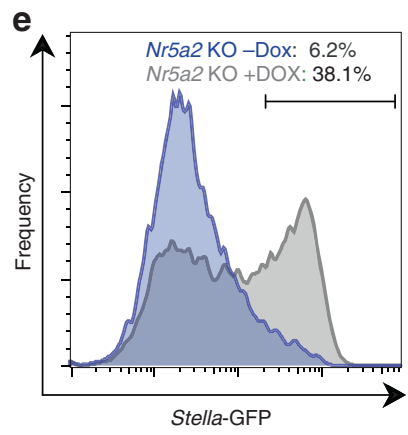

f
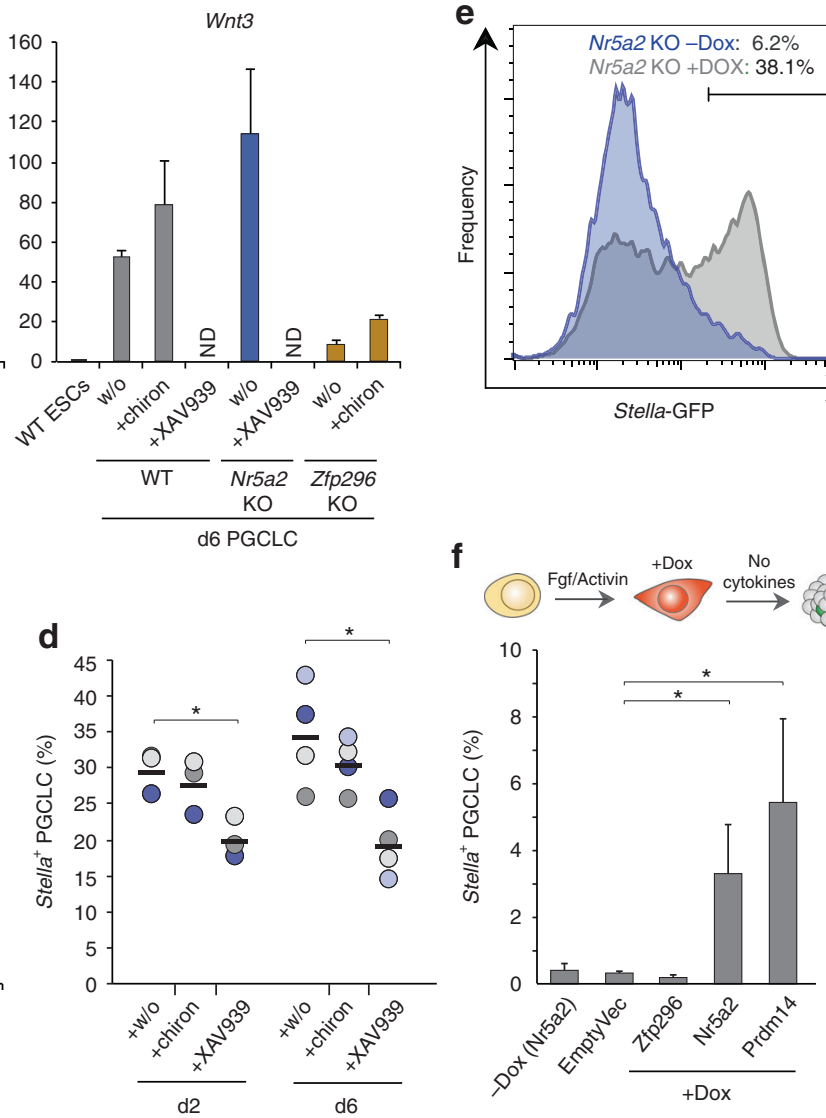

Fig. 6 Role of WNT and rescue of Nr5a2 defects. a Global levels of DNA methylation reprogramming in WT and mutant PGCLC assayed by LUMA. b qRTPCR analysis showing expression of WNT genes/targets are affected by WNT inhibitor (+XAV939) or agonist (+chiron), similarly to knockout PGCLC. W/ o: control without WNT modulation. c Expression of Nanog and mesendoderm gene Hey1 by qRT-PCR in WT and mutant PGCLC with WNT modulation. $\mathbf{d}$ Effects of WNT manipulation on efficiency of PGCLC ontogeny in quadruplicate independent experiments. Bar indicates mean value and ${ }^{*} p<0.05$. e FACS plot showing the Nr5a2-mutant PGCLC specification defect at d2 is rescued by Dox-inducible activation of exogenous Nr5a2 cDNA. Dox is added after EpiLC induction. f Percentage Stella ${ }^{+}$PGCLC induced without cytokines from WT cells upon DOX-inducible expression of the indicated cDNA

generally, our data places Nr5a2 and Zfp296 as critical regulators that provide robustness to germline specification by modulating appropriate WNT activity.

\section{Discussion}

Our study outlines a principle for using CRISPR screens to deconvolve the genetic basis of successive cell fate decisions. By establishing a 'traffic-light' SGET reporter system we traced the pathway from pluripotency to germ cell fate, and identified important genes for each transition. This strategy can be transposed to interrogate multi-step transitions towards any lineage for which a faithful compound reporter or isolation method can be developed.

Utilising SGET we identified genes involved in exiting naive pluripotency, including the previously described key regulators $Z f p 281$ and $T c f 3^{34,35}$, and further validated roles for novel candidates Zmym2, FoxP1 and Uchl5. Among these Zmym2 expression is anti-correlated with naive pluripotency genes at the single-ESC level ${ }^{36}$, and is upregulated when cells are induced to egress from naive pluripotency both in vitro and in vivo (Supplementary Fig 3). Indeed, ZMYM2 is known to directly interact with NANOG ${ }^{37}$ where its negative regulatory activity could blunt NANOG function, or it could alternatively function in this role as part of the LSD1 complex ${ }^{38}$. Interestingly the transcription regulator FoxP1 is also strongly activated during EpiLC induction and, while it can be alternatively spliced to generate a propluripotency isoform ${ }^{39}$, our data implies canonical Foxp1 isoforms play a role in antagonising naive pluripotency. The potential mechanism of function for the deubiquitinase Uchl5 is less clear, but it has previously been associated with regulation of the TGF- $\beta /$ Smad pathway ${ }^{40}$. Future work will be important to characterise the regulatory role of the full repertoire of candidates in control of pluripotent status.

We further exploited the SGET strategy to identify Zfp296 and $\mathrm{Nr} 5 \mathrm{a} 2$ as key functional genes for the germline lineage and as important parts of an expanded PGC gene regulatory network. Deletion of either Nr5a2 or Zfp296 converges on mis-regulation of the WNT pathway, resulting in its aberrant activation or inhibition, respectively, implying that precisely regulated levels of WNT is fundamental for germ cell ontogeny. Indeed, loss of either gene leads to severely disrupted PGCLC development. Of note Nr5a2 is a reported WNT target ${ }^{41}$ and directly interacts with $\beta$-catenin ${ }^{42}$, raising the possibility it forms a negative-feedback loop with WNT to modulate appropriate signalling levels in PGCs. This must strike a balance between WNT-mediated activation of germ cell regulators such as Blimp1 and $P r d m 14^{5}$, and the capacity to subsequently repress the WNT co-activated mesodermal programme. By blunting WNT activity, NR5A2 may ensure the threshold is tipped towards reversibility, since in its 
absence PGCLC development is impaired and linked with aberrant activation of mesendodermal genes. Functionally, Nr5a2 was previously shown to be important for reprogramming somatic cells to iPSC but not for pluripotency per se $\mathrm{e}^{43-45}$. Similarly, PGC undergo reprogramming but do not acquire pluripotency ${ }^{4}$, supporting a role for $\mathrm{Nr} 5 \mathrm{a} 2$ in resetting PGCs away from somatic fates. Notably, NR5A2-binding motifs are among the most significantly enriched within germline-associated promoters ${ }^{46}$, consistent with a direct functional role for Nr5a2 on PGC genes.

In contrast to Nr5a2, loss of Zfp296 results in failure to appropriately upregulate WNT in the germline, as well as affecting some TGF- $\beta$ targets such as Leftyl. Underactive WNT in Zfp296-mutant PGCLC manifests as impaired activation of early germline genes, including Blimp1 and Prdm14. This in turn leads to dramatic loss of PGCLC. Indeed, a recent report has shown the absence of ZFP296 leads to sterility, confirming the long-term consequences of its loss-of-function on the germline ${ }^{32}$. The molecular mechanism through which Zfp296 impacts WNT to regulate PGC ontogeny is an important topic for future research. ZFP296 can, however, influence global H3K9 methylation and has been reported to negatively regulate KLF4 activity ${ }^{32,47}$. The latter counteracts murine Blimp1 expression ${ }^{48}$, implying Zfp296 could contribute to the PGC programme through multiple regulatory routes. Of note, both $Z f p 296$ and Nr5a2 are strongly activated in nascent human PGC (Supplementary Fig 4) ${ }^{49}$, comparably to their upregulation in mouse PGC, indicating they may have a broad conserved role in mammalian germline development.

Our approach and experimental design is both objective and based on functional outcomes, thereby delineating an alternative strategy to identify critical components of cell-type specific gene regulatory networks, which in the case of germ cells, has previously been challenging. The approach preferentially identifies intrinsic cell fate regulators however; not weak-acting or extrinsic regulators, for at least two reasons. First, the addition of extrinsic signalling at high levels (FGF and ACTIVIN for EpiLC; BMP among others for PGCLC) overcomes weak- or signalling receptor-specific effects by acting on multiple redundant pathways to drive fate transitions. Second, the pooled screen strategy means that knockout cells for a given extrinsically acting gene (e.g. Wnt3) can be rescued by paracrine effects from adjacent cells with wild-type alleles. For these reasons, FGF signalling and WNT components were not identified in the exit from pluripotency and PGCLC screens, respectively. Nevertheless, we identified multiple cell-intrinsic candidates. Indeed, recent optimisations to loss-of-function CRISPR libraries ${ }^{50,51}$ will minimise false negatives in future iterations, which can arise due to ineffective gRNAs confounding significance scores, as we observe for Prdm14 for example. In summary, we have used CRISPR screening to identify key regulators for successive cell fate transitions out of naive pluripotency towards the germ cell lineage. In doing so we characterised Zfp296 and Nr5a2 as crucial for driving germ cell development by acting to regulate appropriate WNT levels, thereby controlling the balance between activation of PGC genes and silencing of the somatic programme.

\section{Methods}

ESC culture and PGCLC induction. ESCs were maintained in N2B27 medium supplemented with 2i (PD0325901 (1 $\mu \mathrm{M})$ and CHIR99021 (3 $\mu \mathrm{M}$; both Stemgent), $1000 \mathrm{U} / \mathrm{ml}$ LIF (Cambridge Centre for Stem Cell Research) (2i/L) and 1\% Knockout Serum Replacement (KSR) on fibronectin at $37^{\circ} \mathrm{C}$ in a $5 \% \mathrm{CO}_{2}$ humidified chamber. For serum/LIF (S/L) culture, cells were maintained on gelatin in GMEM supplemented with $10 \%$ FCS, L-Glutamine, non-essential amino acids (NEAA), $0.1 \mathrm{mM} \beta$-mercaptoethanol, and $1000 \mathrm{U} / \mathrm{ml} \mathrm{LIF.} \mathrm{Induction} \mathrm{of} \mathrm{EpiLC} \mathrm{and}$ PGCLC was carried out as previously described ${ }^{12}$. Briefly, ESCs cultured in $2 \mathrm{i} / \mathrm{L}$ were washed thrice with PBS and seeded onto fibronectin $(16.7 \mathrm{mg} / \mathrm{ml})$ coated plates with EpiLC medium (N2B27, 1\% KSR, bFGF (12 ng/ml), Activin A (20 ng/ $\mathrm{ml})$ ) for $42 \mathrm{~h}$. Media was changed every day. EpiLC were then gently dissociated and seeded at 3000 cells per embryoid in ultra low-cell binding U-bottom 96-well plates (NUNC) with PGCLC induction medium (GK15: GMEM, 15\% KSR, NEAA $1 \mathrm{mM}$ sodium pyruvate, $0.1 \mathrm{mM} \beta$-mercaptoethanol, $100 \mathrm{U} / \mathrm{ml}$ penicillin, $0.1 \mathrm{mg} /$ $\mathrm{ml}$ streptomycin and $2 \mathrm{mM}$ L-glutamine; supplemented with cytokines: BMP4 (500 $\mathrm{ng} / \mathrm{ml})$, LIF $(1000 \mathrm{U} / \mathrm{ml})$, SCF $(100 \mathrm{ng} / \mathrm{ml})$, BMP8a $(500 \mathrm{ng} / \mathrm{ml})$ and EGF $(50 \mathrm{ng} /$ $\mathrm{ml})$ ). For large scale (CRISPR screen) PGCLC inductions ( $>4000$ embryoids per replicate), $30 \mu \mathrm{l}$ of PGCLC medium was used for each embryoid, with $20 \mu \mathrm{l}$ additional PGCLC medium added on d2. For smaller-scale PGCLC inductions, $150 \mu \mathrm{l}$ of PGCLC medium was used per embryoid and another $50 \mu \mathrm{l}$ of PGCLC medium was added on $\mathrm{d} 2$. To minimise evaporation, only the inner 60 wells were used during the screen and $200 \mu \mathrm{l}$ of PBS was added to the outer 36 wells. After 2-6 days, EBs were collected for FACS or gene expression analyses. SGET EpiLC were also seeded in GK15 medium without cytokines as a negative control to normalise for background levels of SGET fluorescence in embryoids. Where indicated, WNT inhibitor XAV939 $(10 \mu \mathrm{M})$ or agonist CHIR99021 $(3 \mu \mathrm{M})$ were added upon induction of PGCLC in embryoids. Where indicated DOX was added at $200 \mathrm{ng} / \mu \mathrm{l}$ upon induction of PGCLC in embryoids $+/$ - above mentioned cytokines.

Generation and validation of the SGET reporter. Embryonic stem cell lines carrying Stella-GFP BAC (SG) ${ }^{52}$ were re-derived as previously described, and routinely checked for mycoplasma-negative status using the mycoalert detection kit (Lonza). Low-passage SG lines were used to target an in-frame tdTomato cassette heterozygously into exon 1 of Esg1 (ET) by homologous recombination, thereby generating SGET ESC. Targeting vector(s) for Esg1 were constructed by amplifying homology arms from genomic DNA of mouse ESC by PCR using PrimeSTAR MAX (Takara Bio, Otsu, Japan), tdTomato from ptdTomato-N1 (Takara Bio, Otsu, Japan) and a destabilised domain (d2) with T2A BSD from

pL1L2_IRESdCherryT2Abla-pAneotk vector (a gift from core facility in Cambridge Stem Cell Institute), and assembled using InFusion HD cloning kit (Takara Bio, Otsu, Japan). For gene targeting, $1 \times 10^{7}$ low-passage $(<$ p10) Stella-GFP ESC suspended in PBS were electroporated with $40 \mu \mathrm{g}$ linearised targeting vector using conditions: $800 \mathrm{~V}, 10 \mathrm{~F} 49$ in Gene Pulser equipment (Bio-Rad). ESCs were selected with $10 \mathrm{mg} / \mathrm{ml}$ blasticidin, and colonies were picked and screened for correct targeting by PCR.

We tested for faithful reporter activity using tetraploid embryo complementation ( E8.5) and chimera formation assays (E12.5). For tetraploid complementation assay, 2-cell stage diploid embryos were collected in M2 medium from the oviduct of mice at E1.5, and washed three times with medium containing $0.01 \%$ polyvinyl alcohol (Sigma), $280 \mathrm{mM}$ Mannitol (Sigma), $0.5 \mathrm{mM}$ Hepes (Sigma) and $0.15 \mathrm{mM} \mathrm{MgSO} 4$ (Sigma). Electrofusion of blastomeres to produce tetraploid embryos was subsequently carried out using a DC pulse $(100 \mathrm{~V} / \mathrm{mm}, 30$ ms, 1 time) followed by application of AC pulses (5 V/mm, $10 \mathrm{~s}$ ) using ECM 200 (BTX, Holliston, MA). Tetraploid embryos were transferred into KSOM medium (Merck Millipore) and cultured for $24 \mathrm{~h}$ for 4 -cell/morula injection. For chimera formation assay, morula stage diploid embryos were collected in M2 medium from the oviduct of mice at E2.5. For micro-manipulation, a piezo-driven micromanipulator (Prime Tech, Tokyo, Japan) was used to drill through the zona pellucida and 5-10 SGET ESC were introduced into the perivitelline space of morula stage tetraploid or diploid embryos. They were cultured to the blastocyst stage and then transferred into the uteri of pseudopregnant recipient MF1 female mice (E2.5). Post-implantation embryos were dissected at $\sim$ E4.5, E6.0, E7.0, E8.5 and E12.5 to analyse GFP and tdTomato expression. Notably these assays also confirm the authentic pluripotent status of our SGET line(s). All husbandry and experiments involving mice were authorised by a UK Home Office Project License $80 / 2637$ and carried out in a Home Office-designated facility.

Lentiviral CRISPR screen. A genome-wide lentiviral CRISPR gRNA library was utilised that contains 87,897 gRNAs targeting 19,150 mouse protein-coding genes $^{21}$, with up to five gRNAs per gene (Addgene: \#50947). The gRNA library was amplified with NEB 10-beta electrocompetent Escherichia coli (NEB) as per the recommended protocol. Briefly, E. coli were transformed via high-efficiency electroporation, to ensure faithful library replication, and incubated at $37^{\circ} \mathrm{C}$ for $1 \mathrm{~h}$ with SOC recovery medium (ThermoFisher) before growing in $500 \mathrm{ml} 2 \mathrm{xTY}(16 \mathrm{~g} / \mathrm{l}$ Tryptone, $10 \mathrm{~g} / \mathrm{l}$ Yeast Extract, $5.0 \mathrm{~g} / \mathrm{l} \mathrm{NaCl})+$ ampicillin $(50 \mu \mathrm{g} / \mathrm{ml})$ medium at 37 ${ }^{\circ} \mathrm{C}$ overnight with $230 \mathrm{rpm}$ shaking. Plasmid was purified from $500 \mathrm{ml}$ bacterial cultures using an endotoxin-free plasmid maxi kit (Qiagen) as per manufacturer's instructions. Faithful replication/amplification of the library was confirmed by Illumina sequencing. For lentiviral production, $4 \times 10^{6} \mathrm{HEK} 293 \mathrm{~T}$ cells cultured in DMEM (Invitrogen), 10\% FCS, L-glutamine and Penicillin-Streptomycin were seeded onto $10 \mathrm{~cm}$ poly-lysine coated plates. The following reagents were prepared in OptiMEM (ThermoFisher) per plate: $10 \mu \mathrm{g}$ CRISPR plasmid library, $10 \mu \mathrm{g}$ VSVG-pcDNA3 (Addgene:\#8454), $10 \mu \mathrm{g}$ pCMV-dR8.2 (Addgene:\#8455) and $30 \mu \mathrm{l}$ of Lipofectamine 2000 (ThermoFisher), and incubated with cells for $5-6 \mathrm{~h}$ at $37^{\circ} \mathrm{C}$ in a $5 \% \mathrm{CO}_{2}$ incubator. Medium was subsequently replaced to include Forskolin (5 $\mu \mathrm{M} / \mathrm{ml}$ ), and viral supernatant was collected after $48 \mathrm{~h}$ and $64 \mathrm{~h}$. Cell debris were removed by centrifugation at $1200 \mathrm{rpm}$ for $15 \mathrm{~min}$ at $4{ }^{\circ} \mathrm{C}$, and the supernatants were filtered with $0.45 \mu \mathrm{M}$ filter. To concentrate the virus supernatant, it was combined with 4 XPEG solution $(320 \mathrm{ml}$ of $50 \%$ PEG $6000,40 \mathrm{ml}$ of $5 \mathrm{M} \mathrm{NaCl}, 20$ 
$\mathrm{ml}$ of $1 \mathrm{M}$ HEPES, adjust $\mathrm{pH}$ to 7.4 and add ddH20 until $500 \mathrm{ml}$ ). The virus/PEG mixture was incubated for $4 \mathrm{~h}$ at $4{ }^{\circ} \mathrm{C}$, centrifuged at $2600 \times g$ for $30 \mathrm{~min}$ at $4{ }^{\circ} \mathrm{C}$, and the supernatant aspirated; the process was repeated twice. The viral titre (multiplicity of infection) was assayed by determining the percentage of cells with viral integration after transduction by flow analysing BFP-positive cells and confirmed by qPCR (for BFP).

For the CRISPR screen we first generated SGET ESC that carried constitutive expression of Cas 9 by introducing a CAG-Cas9-pA cassette into SGET cells via PiggyBac-mediated transposition. Clones were subsequently picked and screened to identify a single-copy integration of Cas 9 , and to confirm functional capacity to direct high-efficiency gRNA-directed DNA indels using a GFP reporter. To introduce the CRISPR library, a total of $5 \times 10^{7}$ SGET-Cas 9 ESC were transduced with lentiviral library using a multiplicity of infection (MOI) of 0.3 , with biological replicates infected on independent occasions (gRNA fold coverage per replicate $146 \times ; 296 \times$ total). Transduced SGET ESC were maintained on fibronectin-coated T175 flasks in N2B27, 2i/LIF and 1\% KSR, with $8 \mathrm{ng} / \mu \mathrm{l}$ of polybrene added during lentiviral infection. Medium was changed $24 \mathrm{~h}$ later and cells were selected with puromycin $(1.2 \mu \mathrm{g} / \mathrm{ml})$ for 5 days. High numbers (>30mio) of transduced ESC were passaged every $2-3$ days at low ratio to ensure the complexity of the gRNA library was maintained. ESC pellets were taken at day 12 for analysis and EpiLC were induced on day 12 post-lentiviral transduction, from which PGCLC were subsequently induced.

To quantify the relative frequencies of integrated gRNAs in each population during cell fate transitions, genomic DNA was isolated from day 12 ESC postlentiviral transduction, from $\mathrm{SG}^{-} \mathrm{ET}^{+}$and $\mathrm{SG}^{+} \mathrm{ET}^{+}$EpiLC populations at $42 \mathrm{~h}$, and from $\mathrm{SG}^{+} \mathrm{ET}^{\text {low }}$ PGCLCs from d6 embryoids, in biological duplicate using the DNeasy Blood \& Tissue kit (Qiagen). Purified genomic DNA was used as template for custom primers that specifically amplify the gRNA region, and include overhanging Illumina adaptors and indexes to allow deep sequencing and multiplexing, respectively (synthesised as Ultramers from IDT) (see oligo tables). Prior to PCR, genomic DNA was sonicated with a Biorupter (Diagenode) for $15 \mathrm{~s}$ on 'LOW' power to improve efficiency of amplification. gRNA sequences were amplified in multiple PCR reactions to enable all isolated DNA to be utilised, each with NEBNext ${ }^{\circledR}$ Q5 HotStart HiFi PCR Master Mix (NEB), $0.2 \mu \mathrm{M}$ of universal forward primer (mix of staggers), $0.2 \mu \mathrm{M}$ of indexed reverse primer and genomic DNA ( $650 \mathrm{ng} / 50 \mu \mathrm{lrnn})$. For amplification from the plasmid vector, $15 \mathrm{ng}$ was used as template for replicate amplifications. The following cycling conditions were used: $95^{\circ} \mathrm{C}$ for $2 \mathrm{~min}$, then between 21 and $26 \mathrm{cycles}$ of $\left(98^{\circ} \mathrm{C}\right.$ for $20 \mathrm{~s}, 62^{\circ} \mathrm{C}$ for 20 $\mathrm{s}$ and $72{ }^{\circ} \mathrm{C}$ for $20 \mathrm{~s}$ ) followed by $72{ }^{\circ} \mathrm{C}$ for $1 \mathrm{~min}$ and hold at $4{ }^{\circ} \mathrm{C}$. The number of cycles was optimised for the point at which PCR products can be first visualised on an agarose gel. The PCR reaction was subsequently purified with AMPure ${ }^{\circledast}$ XP beads (Beckman Coulter) using double size selection to remove primer dimers and genomic DNA. Briefly, 0.55x of AMPure beads were added to PCR reaction $(1 \times)$ and incubated for $5 \mathrm{~min}$ at room temperature. The supernatant was collected; the beads contained the unwanted larger fragments and were discarded. An additional $0.3 \times$ of AMPure beads was subsequently added to the supernatant $(1 \times)$ and incubated for $5 \mathrm{~min}$ at room temperature. The supernatant was removed and beads washed twice with $200 \mu \mathrm{l}$ of $80 \% \mathrm{EtOH}$, air dried for $5 \mathrm{~min}$ and DNA was eluted from AMPure beads with EDTA-low TE buffer. The concentration of adaptorligated gRNA amplicons was measured with the Qubit DNA Assay kit (ThermoFisher Scientific) and the fragment distribution determined with an Agilent D1000 ScreenTape System (Agilent Technologies). Libraries were subsequently multiplexed and sequenced with an Illumina HiSeq 2500 using singleend $50 \mathrm{bp}$ reads. As the sequences for all samples were identical up to the gRNA region, these types of low complexity libraries can produce low quality data. To counteract this, forward staggered primers were introduced at equal ratios during PCR amplification, generating offset reads. In addition, libraries were sequenced with four dark cycles and low density $(70 \%)$ clustering.

Gene editing in ESC cell lines. Targeted knockout ESC lines were generated using CRISPR genome editing by either deleting a critical exon to generate a frameshifted null-allele or via inducing frame-shifting null indels into early coding exonic sequences. In brief, 20nt gRNAs encoding complementary sequences to the region/gene to be targeted were cloned into px459 (v2.0) (Addgene:\#62988) using the Bbs1 sites and transfected into SGET ESC with lipofectamine 2000 (ThermoFisher), according to the manufacturers guidelines. A table of the gene targeting gRNAs and the editing strategy is shown below. Transfection of ESC was selected for with $1.2 \mu \mathrm{g} / \mathrm{ml}$ puromycin for $48 \mathrm{~h}$, and ESC were subsequently passaged into 6well plates at low density (1000-5000 ESC per well). One week later colonies were picked and expanded before undergoing genotyping by amplicon sizing or using the tracking of indels by decomposition tool (https://tide.nki.nl). Sanger sequencing on selected clones was subsequently used to confirm the precise mutant sequence of each allele. Multiple mutant lines were catalogued and validated before use in functional assays, where WT and/or heterozygous clones derived from the same targeting process were utilised as control, along with the parental SGET line. For doxycycline-inducible expression, $Z f p 296, N r 5 a 2$ or Prdm 14 cDNA was cloned into a custom PiggyBac vector downstream of a DOX-responsive promoter, and transfected into SGET ESC in conjunction with the reverse transactivator (TET$3 \mathrm{G})$. Genomic integration was selected for with $250 \mu \mathrm{g} / \mathrm{ml}$ neomycin.
Flow cytometry. For FACS, cultured cells or embryoids were dissociated into single cells with TrypLE, and suspended in phosphate buffered saline (PBS) with $0.5 \%$ BSA or $3 \%$ FBS. Filtered cell suspensions were subsequently flow sorted using a Sony SH800Z or a MoFlow high-speed cell sorter (Beckman Coulter) or analysed with BD LSRFortessa X-20 (BD Biosciences). For exit from pluripotency analyses, cells were sorted/analysed based on absence or presence of Stella-GFP expression, while maintaining Esg1-tdTomato activation (SG+/-ET+). For PGCLC, cells were sorted/analysed based on Stella-GFP re-activation coupled with low Esg1-tdTomato. The threshold(s) levels for sorting was set and normalised based on expression levels in pre-optimised SGET ESC. A negative population of ESC without any fluorescence was used to set absolute thresholds. Forward and side scatters were used to gate for the cell population and doublets. For each sort the maximum number of cells was collected, while for flow analysis at least 10,000 cell datapoints were captured to achieve a representative sample.

RNA-seq and gene expression. For RNA-seq of $\mathrm{Nr}^{2} \mathrm{a2} 2^{-/-}, \mathrm{Zfp} 296^{-/-}$and matched WT control cell lines, EpiLC, d2 or d6 PGCLC were dissociated into single-cell solutions with TrypLE and sorted with a Sony SH800Z or a MoFlow high-speed cell sorter (Beckman Coulter) based on appropriate Stella-GFP and Esg1-tdTomato expression. Samples were collected in $150 \mu$ of extraction buffer from the PicoPure RNA isolation kit (Life Technologies) and rapidly frozen on dry ice or liquid nitrogen. Total RNA was subsequently extracted using the PicoPure RNA isolation Kit, including a 15 min on-column DNaseI digestion. RNA integrity number was assessed with RNA HS ScreenTape (Agilent), and all samples confirmed to have a RIN > 8.5. $100 \mathrm{ng}$ of total RNA from triplicate biological independent experiments was used as input for the NEBNext Ultra RNA library Prep Kit for Illumina ${ }^{\circledR}$ (NEB), with the library generated as per manufacturer's instructions. During the final PCR stage, 15 cycles of amplification were performed to generate the adaptor ligated, fragmented cDNA for sequencing. Samples were assessed using the High Sensitivity D1000 ScreenTape assay (Agilent) to ensure the library did not contain primer-dimer contamination after last round of AMPure beads cleaning. The Qubit dsDNA HS assay kit (ThermoFisher Scientific) and the NEBNext Library Quant Kit for Illumina ${ }^{\circledR}$ were used to accurately quantify the concentration of each library preparation. Samples were multiplexed with 12 indexes per lane and a total of two lanes of sequencing on an Illumina HiSeq2500 or HiSeq4000, single-end $50 \mathrm{bp}$, with an average depth of approximately 20 million reads per sample.

For RNA-seq from CRISPR screen samples, 50 ng of total RNA was used as input for the Ovation RNA-seq System v2 (Nugen) as per manufacturer's instructions. Amplified double-stranded (ds) cDNA was diluted into EDTA-low TE (Agilent) and sheared into 230 bp in length using S220 Focused-Ultrasonicator (Covaris) using settings: duty factor $10 \%$, cycle burst 200 , intensity 5 , temp at $4{ }^{\circ} \mathrm{C}$ and treatment time of $5 \mathrm{~min}$ per sample. A high Sensitivity D1000 ScreenTape assay (Agilent) was used to assess the efficiency of library fragmentation. Fragmented ds-cDNA was concentrated with Qiagen Reaction Clean Up kit (MiniElute) and $1 \mu \mathrm{g}$ of the fragmented ds-cDNA used as input for the library preparation using Encore Rapid DR Multiplex Library System (Nugen). This kit ligated the adaptors to repaired-end ds-cDNA without amplification, to mimimise biases introduced during PCR amplification. The KAPA Library Quantification Kit (Kapa bioscience) was used to quantify the concentration of each adaptor-ligated libraries prior to multiplexing. The ESC, EpiLC and PGCLC RNA sequencing libraries were generated in parallel for each replicate, and the biological replicates were generated on independent occasions. Samples were multiplexed and sequenced with Illumina HiSeq1500, single-end 50 bp read length with a minimum depth of $\sim 15$ million reads per sample.

DNA methylation. Global DNA methylation levels were determined using the LUminometric Methylation Analysis (LUMA) method ${ }^{53}$ and bisulfite pyrosequencing. Briefly, genomic DNA was isolated from purified PGCLC using the DNeasy Blood \& Tissue kit (Qiagen) and treated with RNase. 50-100 ng of DNA was digested with MspI/EcoRI and HpaII/EcoRI (NEB) and the subsequent methylation-sensitive overhangs were quantified by Pyrosequencing (PyroMark Q24 Advance) with the dispensation order: GTGTGTCACACAGTGTGT. Global CpG methylation levels were determined from relative peak heights at position(s) $7,8,13$ and 14 using the formula: $\left[\left(2^{*}\left(\mathrm{p} 7^{\star} \mathrm{p} 13\right)\right) /(\mathrm{p} 8+\mathrm{p} 14)\right]^{\mathrm{HpaII} /\left[\left(2^{*}\left(\mathrm{p} 7^{*} \mathrm{p} 13\right)\right) /\right.}$ $(\mathrm{p} 8+\mathrm{p} 14)]^{\mathrm{MspI}}$. CpG methylation at LINE1 loci was determined by bisulfite pyrosequencing using the EpiTect bisulfite kit (Qiagen). PCR amplification and assay design were performed as previously described ${ }^{54}$.

Bioinformatics. For RNA-seq, expression reads were quality-trimmed using TrimGalore to remove adaptors, and aligned to the mouse reference genome (GRCm38/mm10) using TopHat2 guided by ENSEMBL gene models. Mapped reads were imported into SeqMonk analysis software, normalised, and quantitated using the RNA-seq quantitation pipeline. Differentially expressed genes (DEG) were identified using the DESeq2 statistical package with $f d r$ significance thresholds set as $p<0.05$, filtering for a fold-change (FC) $>2$ and mean RPKM expression $>1$ in at least one sample. Analysis of gene ontology enrichment was performed using DEG datasets in the DAVID bioinformatics resource v6.7 (https://david.ncifcrf. gov) and/or the REVIGO visualisation tool (http://revigo.irb.hr). Heatmaps and 
plots were generated using custom scripts in the R statistical package. For analysis of the CRISPR screen, demultiplexed gRNA sequences were extracted from Illumina reads using custom scripts to account for staggered start positions. These were quantitated and statistically analysed using model-based analysis of genomewide CRISPR-Cas9 knockout (MAGeCK) v.0.5.2 software in python ${ }^{22}$. Library sequences were trimmed to exclude the bottom $1 \%$ of reads from the initial library, which primarily corresponded to zero-count gRNAs. Fold-changes in gRNA frequency were calculated as the logarithmic of the mean fold-change between each gRNA set(s) that target a common gene between paired fate transitions. Statistical analysis to identify significantly affected genes was performed using a 'total' normalisation method in MAGeCK to compare gRNA counts with the preceding cell population during ESC-EpiLC-PGCLC transit (or with the vector library in the case of ESC). We subsequently applied the relative ranking algorithm, taking genes scoring $p<0.01$ for negative or positive enrichment in both independent replicate screens as candidates for further validation.

\section{Data availability}

All raw sequencing data related to this study has been deposited in the gene expression omnibus (GEO) repository under accession GSE117473. Other relevant data and reagents that support the findings of this study are available from the corresponding author(s) on reasonable request. Information on oligonucleotide sequences can be found in the supplementary material.

Received: 5 February 2018 Accepted: 8 August 2018

Published online: 16 October 2018

\section{References}

1. Ohinata, Y. et al. A signaling principle for the specification of the germ cell lineage in mice. Cell 137, 571-584 (2009).

2. Lawson, K. A. \& Hage, W. J. Clonal analysis of the origin of primordial germ cells in the mouse. Ciba Found. Symp. 182, 68-84 (1994). discussion 84-91.

3. Saitou, M. \& Yamaji, M. Germ cell specification in mice: signaling transcription regulation, and epigenetic consequences. Reproduction 139, 931-942 (2010).

4. Hackett, J. A., Zylicz, J. J. \& Surani, M. A. Parallel mechanisms of epigenetic reprogramming in the germline. Trends Genet. 28, 164-174 (2012).

5. Aramaki, S. et al. A mesodermal factor, T, specifies mouse germ cell fate by directly activating germline determinants. Dev. Cell 27, 516-529 (2013).

6. Nakaki, F. et al. Induction of mouse germ-cell fate by transcription factors in vitro. Nature 501, 222-226 (2013).

7. $\mathrm{Tu}, \mathrm{S}$. et al. Co-repressor CBFA2T2 regulates pluripotency and germline development. Nature 534, 387-390 (2016).

8. Nady, N. et al. ETO family protein Mtgrl mediates Prdm14 functions in stem cell maintenance and primordial germ cell formation. eLife 4, e10150 (2015).

9. Tang, W. W., Kobayashi, T., Irie, N., Dietmann, S. \& Surani, M. A. Specification and epigenetic programming of the human germ line. Nat. Rev. Genet. 17, 585-600 (2016)

10. Kurimoto, K. et al. Complex genome-wide transcription dynamics orchestrated by Blimp1 for the specification of the germ cell lineage in mice. Genes Dev. 22, 1617-1635 (2008).

11. Yamaji, M. et al. Critical function of Prdm14 for the establishment of the germ cell lineage in mice. Nat. Genet. 40, 1016-1022 (2008).

12. Hayashi, K., Ohta, H., Kurimoto, K., Aramaki, S. \& Saitou, M. Reconstitution of the mouse germ cell specification pathway in culture by pluripotent stem cells. Cell 146, 519-532 (2011).

13. Saitou, M. \& Miyauchi, H. Gametogenesis from pluripotent stem cells. Cell Stem Cell 18, 721-735 (2016).

14. Buecker, C. et al. Reorganization of enhancer patterns in transition from naive to primed pluripotency. Cell Stem Cell 14, 838-853 (2014).

15. Zylicz, J. J. et al. Chromatin dynamics and the role of G9a in gene regulation and enhancer silencing during early mouse development. Elife 4, e09571 (2015).

16. Hikabe, O. et al. Reconstitution in vitro of the entire cycle of the mouse female germ line. Nature 539, 299-303 (2016).

17. Joung, J. et al. Genome-scale CRISPR-Cas 9 knockout and transcriptional activation screening. Nat. Protoc. 12, 828-863 (2017)

18. Han, K. et al. Synergistic drug combinations for cancer identified in a CRISPR screen for pairwise genetic interactions. Nat. Biotechnol. 35, 463-474 (2017).

19. Parnas, O. et al. A genome-wide CRISPR screen in primary immune cells to dissect regulatory networks. Cell 162, 675-686 (2015).

20. Hackett, J. A. et al. Germline DNA demethylation dynamics and imprint erasure through 5-hydroxymethylcytosine. Science 339, 448-452 (2013).
21. Koike-Yusa, H., Li, Y., Tan, E. P., Velasco-Herrera Mdel, C. \& Yusa, K. Genome-wide recessive genetic screening in mammalian cells with a lentiviral CRISPR-guide RNA library. Nat. Biotechnol. 32, 267-273 (2014).

22. $\mathrm{Li}, \mathrm{W}$. et al. MAGeCK enables robust identification of essential genes from genome-scale CRISPR/Cas9 knockout screens. Genome Biol. 15, 554 (2014).

23. Hackett, J. A. \& Surani, M. A. Regulatory principles of pluripotency: from the ground state up. Cell. Stem. Cell. 15, 416-430 (2014).

24. Dunn, S. J., Martello, G., Yordanov, B., Emmott, S. \& Smith, A. G. Defining an essential transcription factor program for naive pluripotency. Science 344, 1156-1160 (2014)

25. Merkle, F. T. et al. Human pluripotent stem cells recurrently acquire and expand dominant negative P53 mutations. Nature 545, 229-233 (2017).

26. Martello, G. et al. Esrrb is a pivotal target of the Gsk3/Tcf3 axis regulating embryonic stem cell self-renewal. Cell Stem Cell 11, 491-504 (2012).

27. Fidalgo, M. et al. Zfp281 functions as a transcriptional repressor for pluripotency of mouse embryonic stem cells. Stem Cells 29, 1705-1716 (2011).

28. Jackson, M. et al. Severe global DNA hypomethylation blocks differentiation and induces histone hyperacetylation in embryonic stem cells. Mol. Cell Biol. 24, 8862-8871 (2004).

29. Yamada, Y., Aoki, H., Kunisada, T. \& Hara, A. Rest promotes the early differentiation of mouse ESCs but is not required for their maintenance. Cell Stem Cell 6, 10-15 (2010).

30. Pare, J. F. et al. The fetoprotein transcription factor (FTF) gene is essential to embryogenesis and cholesterol homeostasis and is regulated by a DR4 element. J. Biol. Chem. 279, 21206-21216 (2004).

31. Takahashi, K. \& Yamanaka, S. Induction of pluripotent stem cells from mouse embryonic and adult fibroblast cultures by defined factors. Cell 126, 663-676 (2006).

32. Matsuura, T., Miyazaki, S., Miyazaki, T., Tashiro, F. \& Miyazaki, J. I. Zfp296 negatively regulates $\mathrm{H} 3 \mathrm{~K} 9$ methylation in embryonic development as a component of heterochromatin. Sci. Rep. 7, 12462 (2017).

33. Fischedick, G. et al. Zfp296 is a novel, pluripotent-specific reprogramming factor. PLoS ONE 7, e34645 (2012).

34. Fidalgo, M. et al. Zfp281 mediates Nanog autorepression through recruitment of the NuRD complex and inhibits somatic cell reprogramming. Proc. Natl Acad. Sci. USA 109, 16202-16207 (2012).

35. Guo, G., Huang, Y., Humphreys, P., Wang, X. \& Smith, A. A PiggyBac-based recessive screening method to identify pluripotency regulators. PLoS ONE 6 , e18189 (2011)

36. Kumar, R. M. et al. Deconstructing transcriptional heterogeneity in pluripotent stem cells. Nature 516, 56-61 (2014).

37. Wang, J. et al. A protein interaction network for pluripotency of embryonic stem cells. Nature 444, 364-368 (2006).

38. Whyte, W. A. et al. Enhancer decommissioning by LSD1 during embryonic stem cell differentiation. Nature 482, 221-225 (2012).

39. Gabut, M. et al. An alternative splicing switch regulates embryonic stem cell pluripotency and reprogramming. Cell 147, 132-146 (2011).

40. Wicks, S. J. et al. The deubiquitinating enzyme UCH37 interacts with Smads and regulates TGF-beta signalling. Oncogene 24, 8080-8084 (2005).

41. Wagner, R. T., Xu, X., Yi, F., Merrill, B. J. \& Cooney, A. J. Canonical Wnt/ beta-catenin regulation of liver receptor homolog-1 mediates pluripotency gene expression. Stem Cells 28, 1794-1804 (2010).

42. Botrugno, O. A. et al. Synergy between LRH-1 and beta-catenin induces G1 cyclin-mediated cell proliferation. Mol. Cell 15, 499-509 (2004).

43. Aaronson, Y., Livyatan, I., Gokhman, D. \& Meshorer, E. Systematic identification of gene family regulators in mouse and human embryonic stem cells. Nucleic Acids Res. 44, 4080-4089 (2016).

44. Guo, G. \& Smith, A. A genome-wide screen in EpiSCs identifies Nr5a nuclear receptors as potent inducers of ground state pluripotency. Development 137, 3185-3192 (2010)

45. Wang, W. et al. Rapid and efficient reprogramming of somatic cells to induced pluripotent stem cells by retinoic acid receptor gamma and liver receptor homolog 1. Proc. Natl Acad. Sci. USA 108, 18283-18288 (2011).

46. $\mathrm{Ng}, \mathrm{J}$. H. et al. In vivo epigenomic profiling of germ cells reveals germ cell molecular signatures. Dev. Cell. 24, 324-333 (2013)

47. Fujii, Y., Kakegawa, M., Koide, H., Akagi, T. \& Yokota, T. Zfp296 is a novel Klf4-interacting protein and functions as a negative regulator. Biochem. Biophys. Res. Commun. 441, 411-417 (2013).

48. Hackett, J. A., Kobayashi, T., Dietmann, S. \& Surani, M. A. Activation of lineage regulators and transposable elements across a pluripotent spectrum. Stem Cell Rep. 8, 1645-1658 (2017).

49. Tang, W. W. et al. A unique gene regulatory network resets the human germline epigenome for development. Cell 161, 1453-1467 (2015).

50. Doench, J. G. et al. Optimized sgRNA design to maximize activity and minimize off-target effects of CRISPR-Cas9. Nat. Biotechnol. 34, 184-191 (2016). 
51. Tzelepis, K. et al. A CRISPR dropout screen identifies genetic vulnerabilities and therapeutic targets in acute myeloid leukemia. Cell Rep. 17, 1193-1205 (2016).

52. Payer, B. et al. Generation of stella-GFP transgenic mice: a novel tool to study germ cell development. Genesis 44, 75-83 (2006).

53. Karimi, M., Luttropp, K. \& Ekstrom, T. J. Global DNA methylation analysis using the luminometric methylation assay. Methods Mol. Biol. 791, 135-144 (2011).

54. Walter, M., Teissandier, A., Pérez-Palacios, R., Bourc'his, D. An epigenetic switch ensures transposon repression upon dynamic loss of DNA methylation in embryonic stem cells. eLife 5, el1418 (2016).

\section{Acknowledgements}

We thank Andy Riddell, Charles Bradshaw and all members of the M.A.S. and J.A.H labs for discussions and support. Funding for this study came from a Wellcome Trust programme grant (C6946/A14492) and Cancer Research UK/Wellcome Trust (092096) core grants to M.A.S, and from a core European Molecular Biology Laboratory (EMBL) grant to J.A.H. The Newton Trust and a Leverhulme Trust Early Career fellowship supported U.G. The James Baird Fund supported Y.H.

\section{Author contributions}

J.A.H. designed the study, performed experiments and bioinformatics, and wrote the manuscript. Y.H. performed experiments and bioinformatics. U.G. performed experiments and wrote the manuscript. K.H.-G. performed experiments. T.K. performed experiments. M.A.S. supervised the study and wrote the manuscript.

\section{Additional information}

Supplementary Information accompanies this paper at https://doi.org/10.1038/s41467018-06230-0.

Competing interests: The authors declare no competing interests.

Reprints and permission information is available online at http://npg.nature.com/ reprintsandpermissions/

Publisher's note: Springer Nature remains neutral with regard to jurisdictional claims in published maps and institutional affiliations.

(c) (i) Open Access This article is licensed under a Creative Commons Attribution 4.0 International License, which permits use, sharing, adaptation, distribution and reproduction in any medium or format, as long as you give appropriate credit to the original author(s) and the source, provide a link to the Creative Commons license, and indicate if changes were made. The images or other third party material in this article are included in the article's Creative Commons license, unless indicated otherwise in a credit line to the material. If material is not included in the article's Creative Commons license and your intended use is not permitted by statutory regulation or exceeds the permitted use, you will need to obtain permission directly from the copyright holder. To view a copy of this license, visit http://creativecommons.org/ licenses/by/4.0/.

(C) The Author(s) 2018 